\title{
Simple Extensions of Polytopes
}

\author{
Volker Kaibel and Matthias Walter \\ Otto-von-Guericke University Magdeburg
}

September 5, 2018

\begin{abstract}
We introduce the simple extension complexity of a polytope $P$ as the smallest number of facets of any simple (i.e., non-degenerate in the sense of linear programming) polytope which can be projected onto $P$. We devise a combinatorial method to establish lower bounds on the simple extension complexity and show for several polytopes that they have large simple extension complexities. These examples include both the spanning tree and the perfect matching polytopes of complete graphs, uncapacitated flow polytopes for non-trivially decomposable directed acyclic graphs, hypersimplices, and random 0/1-polytopes with vertex numbers within a certain range. On our way to obtain the result on perfect matching polytopes we generalize a result of Padberg and Rao's on the adjacency structures of those polytopes. To complement the lower bounding techniques we characterize in which cases known construction techniques yield simple extensions.
\end{abstract}

\section{Introduction}

In combinatorial optimization, linear programming formulations are a standard tool to gain structural insight, derive algorithms and to analyze computational complexity. With respect to both structural and algorithmic aspects linear optimization over a polytope $P$ can be replaced by linear optimization over any (usually higher dimensional) polytope $Q$ of which $P$ can be obtained as the image under a linear map (which we refer to as a projection). Such a polytope $Q$ (along with a suitable projection) is called an extension of $P$.

Defining the size of a polytope as its number of facets, the smallest size of any extension of the polytope $P$ is known as the extension complexity xc $(P)$ of $P$. It has turned out in the past that for several important polytopes related to combinatorial optimization problems the extension complexity is bounded polynomially in the dimension. One of the most prominent examples is the spanning tree polytope of the complete graph $K_{n}$ on $n$ nodes, which has extension complexity $\mathcal{O}\left(n^{3}\right)[15]$.

After Rothvoß [20] showed that there are 0/1-polytopes whose extension complexities cannot be bounded polynomially in their dimensions, only recently Fiorini, Massar, Pokutta, Tiwary and de Wolf [9] could prove that the extension complexities of some concrete and important examples of polytopes like traveling salesman polytopes cannot be bounded polynomially. Similar results 
have then also been deduced for several other polytopes associated with NPhard optimization problems, e.g., by Avis and Tiwary [1] and Pokutta and van Vyve [18]. Very recently, Rothvoß [19] showed that also the perfect matching polytope of the complete graph (with an even number of nodes) has exponential extension complexity, thus exhibiting the first polytope with this property that is associated with a polynomial time solvable optimization problem.

The first fundamental research with respect to understanding extension complexities was Yannakakis' seminal paper [23] of 1991. Observing that many of the nice and small extensions that are known (e.g., the polynomial size extension of the spanning tree polytope of $K_{n}$ mentioned above) have the nice property of being symmetric in a certain sense, he derived lower bounds on extensions with that special property. In particular, he already proved that both perfect matching polytopes as well as traveling salesman polytopes do not have polynomial size symmetric extensions.

It turned out that requiring symmetry in principle actually can make a huge difference for the minimum sizes of extensions (though nowadays we know that this is not really true for traveling salesman and perfect matching polytopes). For instance, Kaibel, Theis, and Pashkovich [14] showed that the polytope associated with the matchings of size $\lfloor\log n\rfloor$ in $K_{n}$ has polynomially bounded extension complexity although it does not admit symmetric extensions of polynomial size. Another example is provided by the permutahedron which has extension complexity $\Theta(n \log n)$ [12], while every symmetric extension of it has size $\Omega\left(n^{2}\right)[17]$

These examples show that imposing the restriction of symmetry may severely influence the smallest possible sizes of extensions. In this paper, we investigate another type of restrictions on extensions, namely the one arising from requiring the extension to be a non-degenerate polytope. A $d$-dimensional polytope is called simple if every vertex is contained in exactly $d$ facets. We denote by sxc $(P)$ the simple extension complexity, i.e., the smallest size of any simple extension of the polytope $P$.

From a practical point of view, simplicity is an interesting property since it formalizes primal non-degeneracy of linear programs. In addition, large parts of combinatorial/extremal theory of polytopes deal with simple polytopes. Furthermore, as with other restrictions like symmetry, there indeed exist nice examples of simple extensions of certain polytopes relevant in optimization. For instance, generalizing the well-known fact that the permutahedron is a zonotope, Wolsey showed in the late 80's (personal communication) that, for arbitrary processing times, the completion time polytope for $n$ jobs is a projection of an $\mathcal{O}\left(n^{2}\right)$-dimensional cube. The main results of this paper show, however, that for several polytopes relevant in optimization (among them both perfect matching polytopes and spanning tree polytopes) insisting on simplicity enforces very large sizes of the extensions. More precisely, we establish that for the following polytopes the simple extension complexity equals their number of vertices (note that the number of vertices of $P$ is a trivial upper bound for $\operatorname{sxc}(P)$, realized by the extension obtained from writing $P$ as the convex hull of its vertices):

- Perfect matching polytopes of complete graphs (Theorem 7.1)

- Uncapacitated flow polytopes of non-decomposable acyclic networks (Theorem 6.1) 
- (Certain) random 0/1-polytopes (Theorem 3.8)

- Hypersimplices (Theorem 4.1)

Furthermore, we prove that

- the spanning tree polytope of the complete graph with $n$ nodes has simple extension complexity at least $\Omega\left(2^{n-o(n)}\right)$ (Theorem 5.2).

The paper is structured as follows: We first focus on known construction techniques and characterize when reflections and disjunctive programming yield simple extensions (Section 2). We continue with some techniques to bound the simple extension complexity of a polytope from below (Section 3). Then we deduce our results on hypersimplices (Section 4), spanning tree polytopes (Section 5), flow polytopes (Section 6), and perfect matching polytopes (Section 7). The core of the latter part is a strengthening of a result of Padberg and Rao's [16] on adjacencies in the perfect matching polytope (Theorem 7.4), which may be of independent interest.

Let us end this introduction by remarking that the concept of simplicial extensions is not interesting. To see this, observe that any $d$-polytope $Q$ with $N$ vertices has at least $d \cdot N$ facet-vertex incidences since every vertex lies in at least $d$ facets. On the other hand, if $Q$ is simplicial (i.e., all facets are simplices) and has $f$ facets, the number of facet-vertex incidences is equal to $d \cdot f$, proving $f \geq N$. For every polytope $P$ with $N$ vertices, every extension polytope has at least $N$ vertices, and hence the smallest possible simplicial extension polytope of $P$ is the simplex with $N$ vertices.

\section{Constructions}

There are three major techniques for constructing extended formulations, namely dynamic programming, disjunctive programming, and reflections. Extensions based on dynamic programs yield network flow polytopes for acyclic graphs which are not simple in general and also have large simple extension complexities (see Section 6).

In this section we characterize for the other two techniques mentioned above in which cases the produced extensions are simple.

\section{$2.1 \quad$ Reflections}

Let $P=\left\{x \in \mathbb{R}^{n}: A x \leq b\right\}$ be a polytope and let $H_{\leq}=\left\{x \in \mathbb{R}^{n}:\langle a, x\rangle \leq \beta\right\}$ be a halfspace in $\mathbb{R}^{n}$. Denoting by $P_{1}:=P \cap H_{\leq}$the intersection of the polytope with the halfspace and by $P_{2}$ the image of $P_{1}$ under reflection at the boundary hyperplane $H_{=}$of $H_{\leq}$, we call $\operatorname{conv}\left(P_{1} \cup P_{2}\right)$ the reflection of $P$ at $H_{\leq}$. The technique in [13] provides an extended formulation for this polytope.

Proposition 2.1 (Kaibel \& Pashkovich [13]). The polytope $Q$ defined by

$$
Q=\left\{(x, y) \in \mathbb{R}^{n+n}: A y \leq b,\langle a, y\rangle \leq\langle a, x\rangle \leq 2 \beta-\langle a, y\rangle,(x-y) \in \operatorname{span}(a)\right\}
$$

together with the projection onto the $x$-space is an extension of $\operatorname{conv}\left(P_{1} \cup P_{2}\right)$.

Our contribution is the next theorem which clarifies under which circumstances $Q$ is a simple polytope. 
Theorem 2.2. Let $Q$ be the extension polytope for the reflection of $P$ at $H_{\leq}$ as defined in this subsection, let $P_{1}:=P \cap H_{\leq}$, and let $F:=P_{1} \cap H_{=}$be the intersection of $P_{1}$ with the reflection hyperplane.

Then $Q$ is simple if and only if $P_{1}$ is simple and either $P_{1}=F$, or $F$ is a facet of $P_{1}$ or $F=\varnothing$.

Proof. We first observe that the faces

$$
\begin{aligned}
& Q_{1}:=Q \cap\left\{(x, y) \in \mathbb{R}^{n} \times \mathbb{R}^{n}:\langle a, y\rangle=\langle a, x\rangle\right\} \\
& Q_{2}:=Q \cap\left\{(x, y) \in \mathbb{R}^{n} \times \mathbb{R}^{n}:\langle a, x\rangle=2 \beta-\langle a, y\rangle\right\}
\end{aligned}
$$

of $Q$ are both affinely isomorphic to $P_{1}$. Thus $Q$ can only be simple if $P_{1}$ is so. If $P_{1} \subseteq H_{=}$holds, $Q=Q_{1}=Q_{2}$ and $Q$ is simple if and only if $P_{1}$ is simple, proving the equivalence in case $P_{1}=F$. Otherwise, let $d:=\operatorname{dim} P_{1}$ and observe that $\operatorname{dim} Q=d+1$ holds because $Q_{1}$ and $Q_{2}$ are proper faces of $Q$ and $Q$ 's dimension cannot be larger than $d+1$. Furthermore, $(x, y) \in Q_{1} \cap Q_{2}$ holds if and only if $\langle a, x\rangle=\beta$ is satisfied, hence $Q_{1} \cap Q_{2}$ is affinely isomorphic to $F$. Define $k:=\operatorname{dim} F$.

We now assume that $Q$ is simple and $F \neq \varnothing$, i.e., $k \geq 0$ holds. Let $v$ be any vertex of $Q_{1} \cap Q_{2}$. Since $Q$ is simple and of dimension $d+1, v$ has $d+1$ adjacent vertices, $k$ of which lie in $Q_{1} \cap Q_{2}$ (isomorphic to $F$ ). Furthermore, $v$ has $d$ neighbors in $Q_{i}$ for $i=1,2$. Hence, $k$ of these vertices lie in $Q_{1} \cap Q_{2}, d-k$ lie in $Q_{1} \backslash Q_{2}$ and $d-k$ lie in $Q_{2} \backslash Q_{1}$. The resulting equation $k+(d-k)+(d-k)=d+1$ yields $k=d-1$, i.e., $F$ is a facet of $P_{1}$. This proves necessity of the condition.

To prove sufficiency, from now on assume that $P_{1}$ is simple and $\operatorname{dim} Q=d+1$ holds. We now prove that every vertex $(x, y)$ of $Q$ not lying in $Q_{1} \cap Q_{2}$ lies in at most (thus, exactly) $d+1$ facets of $Q$. First, $y$ can satisfy at most $d$ inequalities of $A y \leq b$ with equality because $P_{1}$ is simple. Second, $(x, y)$ can satisfy at most one of the other two inequalities with equality since otherwise, $\langle a, x\rangle=\beta$ would hold, contradicting the fact that $(x, y) \notin Q_{1} \cap Q_{2}$. Hence, the vertex lies in at most $d+1$ facets which proves the claim. This already proves that $Q$ is simple in the case $F=\varnothing$, since then there are no further vertices.

It remains to show that if $F$ is a facet of $P_{1}$ then every vertex $(x, y)$ of $Q_{1} \cap Q_{2}$ has at most $d+1$ neighbors in $Q$. In this case, $Q_{1} \cap Q_{2}$ is a facet of $Q_{1}$ and of $Q_{2}$ which in turn are facets of $Q$. Since $Q_{1} \cap Q_{2}$ is a facet of the simple polytope $Q_{i}$ for $i=1,2$, the vertex $(x, y)$ has $d-1$ neighbors in the (simple) facet $Q_{1} \cap Q_{2}$ and 1 neighbor in $Q_{i} \backslash\left(Q_{1} \cap Q_{2}\right)$. In total, $(x, y)$ has $d+1$ neighbors, because all vertices of $Q$ are vertices of $Q_{1}$ or $Q_{2}$ since for fixed $y$ with $A y \leq b$, any $x$ with $(x-y) \in \operatorname{span}(a)$ must satisfy one of the other two inequalities with equality if it is an extreme point.

An interesting observation is that in case of a reflection at a hyperplane $H_{=}$ which does not intersect the given polytope $P$, the resulting extension polytope is combinatorially equivalent to $P \times[0,1]$. This yields a (deformed) cube if such a reflection is applied iteratively if the initial polytope is a cube. Examples are the extensions of size $2 \log m$ for regular $m$-gons for the case of $m=2^{k}$ with $k \in \mathbb{N}$.

Theorem 2.3. Let $k \in \mathbb{N}$. The simple extension complexity of a regular $2^{k}$-gon is at most $2 k$. 


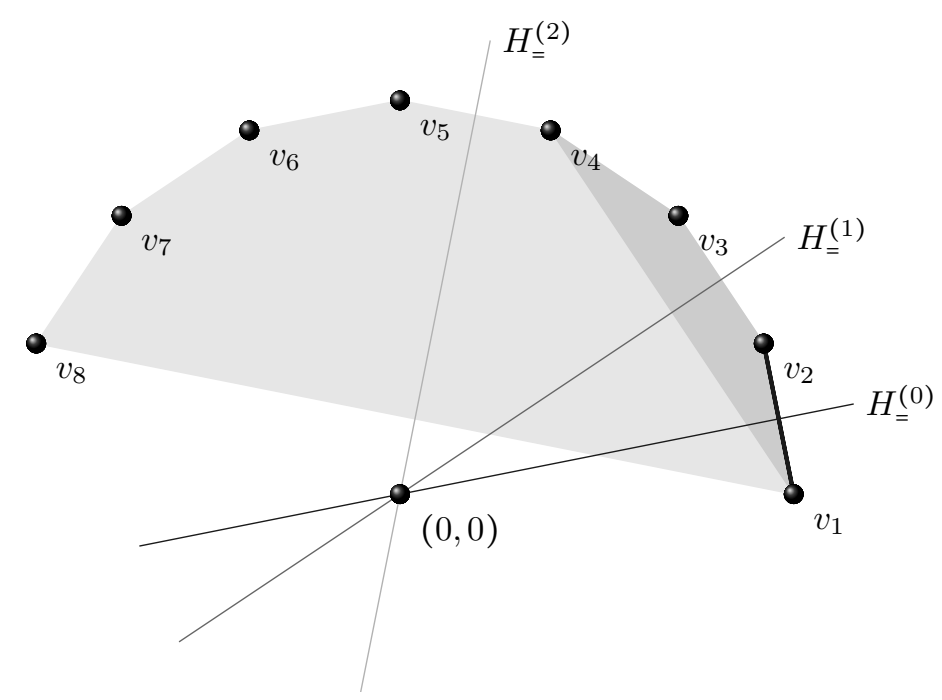

Figure 1: Some Reflections used in the Proof of Theorem 2.3 for a 16-gon.

Proof. We recursively define a series of polytopes as follows: The initial (simple) polytope is $P^{(0)}:=\{(1,0)\}$, i.e., a single point. Since the extensions we construct are located in increasingly higher-dimensional spaces we write coordinates as $(x, y, z) \in \mathbb{R} \times \mathbb{R} \times \mathbb{R}^{*}$, where the dimension of the $z$-space increases, initially being zero.

We now define for $i=0,1,2, \ldots, k-1$ the polytope $P^{(i+1)}$ as the reflection of $P^{(i)}$ at the halfspace

$H_{\leq}^{(i)}:=\left\{(x, y, z) \in \mathbb{R} \times \mathbb{R} \times \mathbb{R}^{*}:-\sin \left(\left(2^{i}-1\right) \cdot \pi / 2^{k}\right) x+\cos \left(\left(2^{i}-1\right) \cdot \pi / 2^{k}\right) y \leq 0\right\}$.

Theorem 3 in [13] shows that $P^{(k)}$ is an extension of a regular $2^{k}$-gon. If we label the vertices of this $2^{k}$-gon with $v_{1}, v_{2}, \ldots, v_{2^{k}}$ in counter-clockwise order starting with $v_{1}=(1,0)$, the proof even shows that the projection of $P^{(i)}$ onto the first two coordinates equals the convex hull of the vertices $v_{1}, v_{2}, \ldots, v_{2^{i}}$. Now for every $i=0,1, \ldots, k-1$, the polytope $P^{(i)}$ does not intersect $H_{=}^{(i)}$ since the projection of such an intersection point would lie outside the mentioned convex hull.

This ensures that by induction all polytopes $P^{(i)}$ for $i=0,1,2, \ldots, k$ are simple by Theorem 2.2 and that the last polytope $P^{(k)}$ is a simple extension of the regular $2^{k}$-gon.

\subsection{Disjunctive Programming}

The third major technique to construct extended formulations is by means of disjunctive programming, introduced by Balas [2],[3]. We only consider the special case of a disjunction of two polytopes $P_{1}, P_{2} \subseteq \mathbb{R}^{n}$ and are interested in an extension of the convex hull of the union of the two.

A helpful tool is the homogenization homog $P$ of a polytope $P$, defined as $\operatorname{homog} P:=\operatorname{cone}(P \times\{1\})$, where cone $(\cdot)$ denotes the conic hull. We say that 
a pointed polyhedral cone $C$ is weakly simple if every extreme ray of $C$ lies in exactly $\operatorname{dim}(C)-1$ facets and strongly simple if $C$ is a simple polyhedron. Clearly, a strongly simple cone is also weakly simple. Furthermore, if we have $C=\operatorname{homog} P$ then $C$ is weakly simple if and only if $P$ is simple and $C$ is strongly simple if and only if $P$ is a simplex. We will need the following lemma about weak simplicity of cartesian products of cones.

Lemma 2.4. Given two pointed polyhedral cones $C_{1} \subseteq \mathbb{R}^{n_{1}}, C_{2} \subseteq \mathbb{R}^{n_{2}}$, their product cone $C:=C_{1} \times C_{2} \subseteq \mathbb{R}^{n_{1}+n_{2}}$ is weakly simple if and only if both $C_{1}$ and $\mathrm{C}_{2}$ are strongly simple.

Proof. It is easy to check that $C_{1} \times C_{2}=\left\{\left(x_{1}, x_{2}\right) \in \mathbb{R}^{n_{1}+n_{2}}: x_{i} \in C_{i} \quad i=1,2\right\}$ is a pointed polyhedral cone again. Furthermore, the faces of $C_{1} \times C_{2}$ are exactly the products of faces of $C_{1}$ and $C_{2}$, their dimensions add up, and a face $F_{1} \times F_{2}$ of $C_{1} \times C_{2}$ is contained in another face $G_{1} \times G_{2}$ if and only if $F_{1} \subseteq G_{1}$ and $F_{2} \subseteq G_{2}$ hold.

Hence, the extreme rays of $C_{1} \times C_{2}$ are either products of extreme rays of $C_{1}$ with $\mathbb{O}_{n_{2}}$ or products of $\mathbb{O}_{n_{1}}$ with extreme rays of $C_{2}$. Similarly, the facets of $C_{1} \times C_{2}$ are either products of facets of $C_{1}$ with $C_{2}$ or products of $C_{1}$ with facets of $C_{2}$.

We consider an extreme ray of $C$, w.l.o.g. of the form $r \times \mathbb{O}_{n_{2}}$, where $r$ is an extreme ray $r$ of $C_{1}$. It is clearly contained in the facets $F_{1} \times C_{2}$ where $F_{1}$ is a facet of $C_{1}$ containing $r$. Now for every facet $F_{2}$ of $C_{2}$, we have $\mathbb{O}_{n_{2}} \subseteq F_{2}$ and hence $C_{1} \times F_{2}$ contains $r \times \mathbb{O}_{n_{2}}$.

Thus, if $r$ is contained in $k$ facets of $C_{1}$ and $C_{2}$ has $\ell$ facets then $r \times \mathbb{O}_{n_{2}}$ is contained in $k+\ell$ facets of $C_{1} \times C_{2}$.

We always have $k \geq \operatorname{dim} C_{1}-1$ and $\ell \geq \operatorname{dim} C_{2}$ since $C_{1}, C_{2}$ are pointed polyhedral cones. Hence, $k+\ell \geq \operatorname{dim} C_{1}+\operatorname{dim} C_{2}-1$ holds and we have equality if and only if $k=\operatorname{dim} C_{1}-1$ and $\ell=\operatorname{dim} C_{2}$ are satisfied, and hence $C_{1}$ and $C_{2}$ are strongly simple.

We now turn to the mentioned extension of $P=\operatorname{conv}\left(P_{1} \cup P_{2}\right)$. Define

$$
Q=\left\{\left(x^{1}, \lambda_{1}, x^{2}, \lambda_{2}\right) \in \operatorname{homog}\left(P_{1}\right) \times \operatorname{homog}\left(P_{2}\right): \lambda_{1}+\lambda_{2}=1\right\} .
$$

It is well-known that $Q$ together with the projection $\left(x^{1}, \lambda_{1}, x^{2}, \lambda_{2}\right) \mapsto x^{1}+x^{2}$ yields an extension of $P$. we now characterize when $Q$ is a simple polytope.

Theorem 2.5. The extension polytope $Q$ of the disjunctive program for the polytope $P=\operatorname{conv}\left(P_{1} \cup P_{2}\right)$ is simple if and only if $P_{1}$ and $P_{2}$ are simplices.

Proof. As $Q$ is the intersection of the pointed cone $C=\operatorname{homog}\left(P_{1}\right) \times \operatorname{homog}\left(P_{2}\right)$ with the hyperplane defined by $\lambda_{1}+\lambda_{2}=1$ (which does not contain any of $C$ 's extreme rays), we know that $Q$ is simple if and only if $C$ is weakly simple. Now Lemma 2.4 yields the result.

\section{Bounding Techniques}

Let $P \subseteq \mathbb{R}^{n}$ be a polytope with $N$ vertices. The faces of $P$ form a graded lattice $\mathcal{L}(P)$, ordered by inclusion (see [24]). 
Clearly, $P$ is the set of all convex combinations of its vertices, immediately providing an extended formulation of size $N$ :

$$
P=\operatorname{proj}_{x}\left\{(x, y) \in \mathbb{R}^{n} \times \mathbb{R}_{+}^{V}: x=\sum_{v \in V} y_{v} v, \sum_{v \in V} y_{v}=1\right\}
$$

Here, $\operatorname{proj}_{x}(\cdot)$ denotes the projection onto the space of $x$-variables and $V$ is the set of vertices of $P$. Note that this trivial extension is simple since the extension is an $(N-1)$-simplex.

An easy observation for extensions $P=\pi(Q)$ with $Q \subseteq \mathbb{R}^{d}$ and $\pi: \mathbb{R}^{d} \rightarrow \mathbb{R}^{n}$ is that the assignment $F \mapsto \pi^{-1}(F) \cap Q=\{y \in Q: \pi(y) \in F\}$ defines a map $j$ which embeds $\mathcal{L}(P)$ into $\mathcal{L}(Q)$, i.e., it is one-to-one and preserves inclusion in both directions (see [8]). Note that this embedding furthermore satisfies $j\left(F \cap F^{\prime}\right)=j(F) \cap j\left(F^{\prime}\right)$ for all faces $F, F^{\prime}$ of $P$ (where the nontrivial inclusion $j(F) \cap j\left(F^{\prime}\right) \subseteq j\left(F \cap F^{\prime}\right)$ follows from $\pi\left(j(F) \cap j\left(F^{\prime}\right)\right) \subseteq \pi(j(F)) \cap \pi\left(j\left(F^{\prime}\right)\right)=$ $\left.F \cap F^{\prime}\right)$. We use the shorthand notation $j(v):=j(\{v\})$ for vertices $v$ of $P$.

We consider the face-vertex non-incidence graph $G_{\mathcal{N}}(P)$ which is a bipartite graph having the faces and the vertices of $P$ as the node set and edges $\{F, v\}$ for all $v \notin F$. Every facet $\hat{f}$ of an extension induces two node sets of this graph in the following way:

$$
\begin{aligned}
& \mathcal{F}(\hat{f}):=\quad\{F \text { face of } P: j(F) \subseteq \hat{f}\} \\
& \mathcal{V}(\hat{f}):=\quad\{v \text { vertex of } P: j(v) \nsubseteq \hat{f}\}
\end{aligned}
$$

We call $\mathcal{F}(\hat{f})$ and $\mathcal{V}(\hat{f})$ the set of faces (resp. vertices) induced by the facet $\hat{f}$ (with respect to the extension $P=\pi(Q)$ ). Typically, the extension and the facet $\hat{f}$ are fixed and we just write $\mathcal{F}$ (resp. $\mathcal{V}$ ). It may happen that $\mathcal{V}(\hat{f})$ is equal to the whole vertex set, e.g., if $\hat{f}$ projects into the relative interior of $P$. If $\mathcal{V}(\hat{f})$ is a proper subset of the vertex set we call facet $\hat{f}$ proper w.r.t. the projection.

For each facet $\hat{f}$ of an extension of $P$ the face and vertex sets $\mathcal{F}(\hat{f}), \mathcal{V}(\hat{f})$ together induce a biclique (i.e., complete bipartite subgraph) in $G_{\mathcal{N}}(P)$. It follows from Yannakakis [23] that every edge in $G_{\mathcal{N}}(P)$ is covered by at least one of those induced bicliques. We provide a brief combinatorial argument for this (in particular showing that we can restrict to proper facets) in the proof of the following proposition.

Proposition 3.1. Let $P=\pi(Q)$ be an extension.

Then the subgraph of $G_{\mathcal{N}}(P)$ induced by $\mathcal{F}(\hat{f}) \cup \mathcal{V}(\hat{f})$ is a biclique for every facet $\hat{f}$ of $Q$. Furthermore, every edge $\{F, v\}$ of $G_{\mathcal{N}}(P)$ is covered by at least one of the bicliques induced by a proper facet.

Proof. Let $\hat{f}$ be one of the facets and assume that an edge $\{F, v\}$ with $F \in \mathcal{F}(\hat{f})$ and $v \in \mathcal{V}(\hat{f})$ is not present in $G_{\mathcal{N}}(P)$, i.e., $v \in F$. From $v \in F$ we obtain $j(v) \subseteq j(F) \subseteq \hat{f}$, a contradiction to $v \in \mathcal{V}(\hat{f})$.

To prove the second statement, let $\{F, v\}$ be any edge of $G_{\mathcal{N}}(P)$, i.e., $v \notin F$. Observe that the preimages $G:=j(F)$ and $g:=j(v)$ are also not incident since $j$ is a lattice embedding. As $G$ is the intersection of all facets of $Q$ it is contained in (the face-lattice of a polytope is coatomic), there must be at least one facet 
$\hat{f}$ containing $G$ but not $g$ (since otherwise $g$ would be contained in $G$ ), yielding $F \in \mathcal{F}(\hat{f})$ and $v \in \mathcal{V}(\hat{f})$.

If $F \neq \varnothing$, any vertex $w \in F$ satisfies $j(w) \subseteq G \subseteq \hat{f}$ and hence $\hat{f}$ is a proper facet. If $F=\varnothing$, let $w$ be any vertex of $P$ distinct from $v$. The preimages $j(v)$ and $j(w)$ clearly satisfy $j(v) \nsubseteq j(w)$. Again, since the face-lattice of $Q$ is coatomic, there exists a facet $\hat{f}$ with $j(w) \subseteq \hat{f}$ but $j(v) \nsubseteq \hat{f}$. Hence, $\hat{f}$ is a proper facet and (since $\varnothing=F \subseteq \hat{f}) F \in \mathcal{F}(\hat{f})$ and $v \in \mathcal{V}(\hat{f})$ holds.

Before moving on to simple extensions we mention two useful properties of the induced sets. Both can be easily verified by examining the definitions of $\mathcal{F}$ and $\mathcal{V}$. See Figure 2 for an illustration.

Lemma 3.2. Let $\mathcal{F}$ and $\mathcal{V}$ be the face and vertex sets induced by a facet of an extension of $P$, respectively. Then $\mathcal{F}$ is closed under taking subfaces and $\mathcal{V}=\left\{v\right.$ vertex of $\left.P: v \notin \bigcup_{F \in \mathcal{F}} F\right\}$ holds.

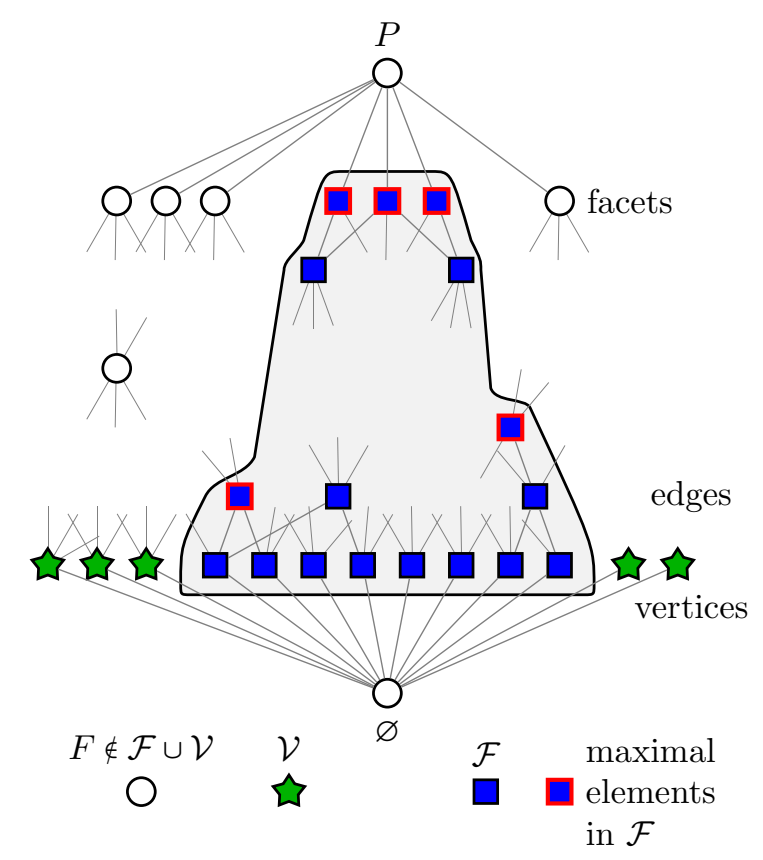

Figure 2: The Sets $\mathcal{F}$ and $\mathcal{V}$ in the Face Lattice.

For the remainder of this section we assume that the extension polytope $Q$ is a simple polytope and that $\mathcal{F}$ and $\mathcal{V}$ are face and vertex sets induced by a facet of $Q$.

Theorem 3.3. Let $\mathcal{F}$ and $\mathcal{V}$ be the face and vertex sets induced by a facet of a simple extension of $P$, respectively. Then

(a) all pairs $\left(F, F^{\prime}\right)$ of faces of $P$ with $F \cap F^{\prime} \neq \varnothing$ and $F, F^{\prime} \notin \mathcal{F}$ satisfy $F \cap F^{\prime} \notin \mathcal{F}$,

(b) the (inclusion-wise) maximal elements in $\mathcal{F}$ are facets of $P$, 
(c) and every vertex $v \notin \mathcal{V}$ is contained in some facet $F$ of $P$ with $F \in \mathcal{F}$.

Proof. Let $\hat{f}$ be the facet of $Q$ inducing $\mathcal{F}$ and $\mathcal{V}$ and $F, F^{\prime}$ two faces of $P$ with non-empty intersection. Since $F \cap F^{\prime} \neq \varnothing$, we have $j\left(F \cap F^{\prime}\right) \neq \varnothing$, thus the interval in $\mathcal{L}(Q)$ between $j\left(F \cap F^{\prime}\right)$ and $Q$ is a Boolean lattice, i.e., isomorphic to the face-lattice of a simplex, (because $Q$ is simple, see Proposition 2.16 in [24]). Suppose $F \cap F^{\prime} \in \mathcal{F}(\hat{f})$. Then $\hat{f}$ is contained in that interval and it is a coatom, hence it contains at least one of $j(F)$ and $j\left(F^{\prime}\right)$ due to $j(F) \cap j\left(F^{\prime}\right)=j\left(F \cap F^{\prime}\right)$. But this implies $j(F) \in \mathcal{F}$ or $j\left(F^{\prime}\right) \in \mathcal{F}$, proving (a).

For (b), let $F$ be an inclusion-wise maximal face in $\mathcal{F}$ but not a facet of $P$. Then $F$ is the intersection of two faces $F_{1}$ and $F_{2}$ of $P$ properly containing $F$. Due to the maximality of $F, F_{1}, F_{2} \notin \mathcal{F}$ but $F_{1} \cap F_{2} \in \mathcal{F}$, contradicting (a).

Statement (c) follows directly from (b) and Lemma 3.2.

In order to use the Theorem 3.3 for deriving lower bounds on the sizes of simple extensions of a polytope $P$, one needs to have good knowledge of parts of the face lattice of $P$. The part one usually knows most about is formed by the vertices and edges of $P$. Therefore, we specialize Theorem 3.3 to these faces for later use.

Let $G=(V, E)$ be a graph and denote by $\delta(W) \subseteq E$ the cut-set of a node-set $W$. Define the common neighbor operator $\Lambda(\cdot)$ by

$$
\Lambda(W):=W \cup\{v \in V: \exists\{u, v\},\{v, w\} \in \delta(W): u \neq w\} .
$$

A set $W \subseteq V$ is then a (proper) common neighbor closed (for short $\Lambda$-closed) set if $\Lambda(W)=W$ (and $W \neq V$ ) holds. We call sets $W$ with a minimum node distance of at least 3 (i.e., the distance-2-neighborhood of a node $w \in W$ does not contain another node $\left.w^{\prime} \in W\right)$ isolated. Isolated node sets are clearly $\Lambda$-closed. Note that singleton sets are isolated and hence proper $\Lambda$-closed. In particular, the vertex sets induced by the facets of the trivial extension (see beginning of Section 3) are the singleton sets.

Using this notion, we obtain the following corollary of Theorem 3.3.

Corollary 3.4. The vertex set $\mathcal{V}$ induced by a proper facet of a simple extension of $P$ is a proper $\Lambda$-closed set.

Proof. Theorem 3.3 implies that for every $\{u, v\},\{v, w\}$ of (distinct) adjacent edges of $P$, we have

$$
\{u, v\},\{v, w\} \notin \mathcal{F} \Rightarrow\{v\} \notin \mathcal{F}
$$

Due to Lemma 3.2, $\mathcal{V}=\{v$ vertex of $P: v \notin \cup \mathcal{F}\}$, where $\mathcal{F}$ is the face set induced by the same facet. Hence, $v \notin \mathcal{V}$ implies $\{u, v\} \in \mathcal{F}$ or $\{v, w\} \in \mathcal{F}$, thus $u \notin \mathcal{V}$ or $w \notin \mathcal{V}$ and we conclude that $\mathcal{V}$ is $\Lambda$-closed.

Furthermore, $\mathcal{V}$ is not equal to the whole vertex set of $P$ since the given facet is proper.

We just proved that every biclique $\mathcal{F} \cup \mathcal{V}$ induced by a (proper) facet from a simple extension must satisfy certain properties. The next example shows that these properties are not sufficient for an extension polytope to be simple. 
Example 3.5. Define $m_{1}, \ldots, m_{7} \in \mathbb{R}^{3}$ to be the columns of the matrix

$$
M:=\left(\begin{array}{ccccccc}
1 & 5 & 1 & 0 & -1 & -5 & -1 \\
1 & 0 & -1 & 0 & 1 & 0 & -1 \\
0 & -4 & 0 & 1 & 0 & -4 & 0
\end{array}\right),
$$

and let $Q:=\operatorname{conv}\left\{m_{1}, \ldots, m_{7}\right\} \subseteq \mathbb{R}^{3}$ be their convex hull. The vertex $m_{4}$ has 4 neighbors, that is, $Q$ is not simple. Let $P$ be the projection of $Q$ onto the first two coordinates. Observe that $P$ is a 6-gon and that the only relevant types of faces $F, F^{\prime}$ are adjacent edges of $P$. It is quickly verified that all induced face and vertex sets satisfy Theorem 3.3 and Corollary 3.4, respectively.

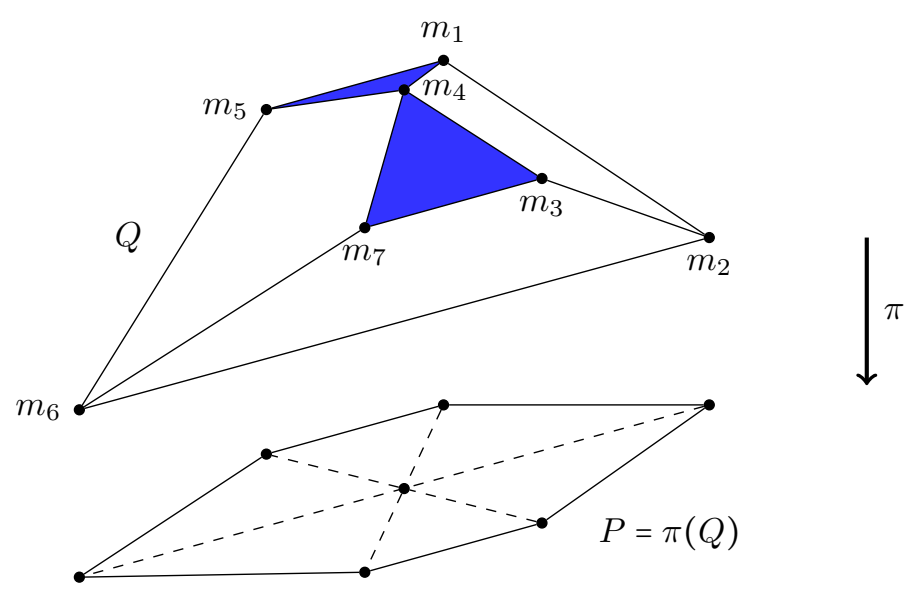

Figure 3: Polytope $Q$ from Example 3.5 and its projection $P$.

Note that this example only shows that we cannot decide from the biclique covering whether the extension is simple. It may still be true that for such biclique coverings there always exists a simple extension.

The polytope $Q$ from the example can be used to show that Corollary 3.4 is indeed a specialization of Theorem 3.3 (a). To see this, consider the set $\mathcal{F}$ of faces consisting of conv $\left\{m_{1}, m_{4}, m_{5}\right\}$, conv $\left\{m_{3}, m_{4}, m_{7}\right\}$ and all their subfaces. Lemma 3.2 implies $\mathcal{V}=\left\{m_{2}, m_{6}\right\}$ which is proper $\Lambda$-closed. But $\mathcal{F}$ does not satisfy Theorem 3.3 (a) for the choice $F:=\operatorname{conv}\left\{m_{1}, m_{2}, m_{3}, m_{4}\right\} \notin \mathcal{F}, F^{\prime}:=$ $\operatorname{conv}\left\{m_{4}, m_{5}, m_{6}, m_{7}\right\} \notin \mathcal{F}$ since $F \cap F^{\prime}=\left\{m_{4}\right\} \in \mathcal{F}$.

Nevertheless we can obtain useful lower bounds from Theorem 3.3 and Corollary 3.4.

Corollary 3.6. The node set of a polytope $P$ can be covered by $\operatorname{sxc}(P)$ many proper $\Lambda$-closed sets.

Lemma 3.7. Let $P$ be a polytope and $G$ its graph. If all proper $\Lambda$-closed sets in $G$ are isolated then the simple extension complexity of $P$ is greater than the maximum size of the neighborhood of any node of $G$.

Proof. Let $w$ be a node maximizing the size of the neighborhood and let $W$ be the neighborhood of $w$. Since no isolated set can contain more than one node from $W \cup\{w\}$, Corollary 3.6 implies the claim. 
Using knowledge about random 0/1-polytopes, we can easily establish the following result.

Theorem 3.8. There is a constant $\sigma>0$ such that a random d-dimensional $0 / 1$-polytope $P$ with at most $2^{\sigma d}$ vertices asymptotically almost surely has a simple extension complexity equal to its number of vertices.

Proof. One of the main results of Gillmann's thesis (See Theorem 3.37 in [11] for $k=2$ ) is that there is such a $\sigma$ ensuring that a random $d$-dimensional $0 / 1$ polytope $P$ with at most $2^{\sigma d}$ vertices asymptotically almost surely has every pair of vertices adjacent. Since in this situation the only proper $\Lambda$-closed sets are the singletons, Corollary 3.6 yields the claim.

\section{$4 \quad k$-Hypersimplex}

Let $\Delta(k)$ denote the $k$-hypersimplex in $\mathbb{R}^{n}$, i.e., the $0 / 1$-cube intersected with the hyperplane $\left\langle\mathbb{1}_{n}, x\right\rangle=k$. Note that its vertices are all $0 / 1$-vectors with exactly $k 1$ 's, since the above linear system is totally unimodular (a row of ones together with two unit matrices). It follows from the knowledge about edges and 2-faces of the cube that two vertices of $\Delta(k)$ are adjacent if and only if they differ in exactly two coordinates. In other words, all neighbors of a vertex $x$ can be obtained by replacing a 1 by a 0 at some index and a 0 by a 1 at some other index. Observe that $\Delta(k)$ is almost simple for $2 \leq k \leq n-2$ in the sense that its dimension is $n-1$, but every vertex lies in exactly $n$ facets. With this in mind, the following result may seem somewhat surprising.

Theorem 4.1. Let $1 \leq k \leq n-1$. The simple extension complexity of $\Delta(k) \subseteq \mathbb{R}^{n}$ is equal to its number of vertices $\left(\begin{array}{l}n \\ k\end{array}\right)$.

Proof. The case of $k=1$ or $k=n-1$ is clear since then $\Delta(k)$ is an $(n-1)$ dimensional simplex.

Let $2 \leq k \leq n-2$ and $\mathcal{F}$ and $\mathcal{V}$ be face and vertex sets induced by a proper facet of a simple extension of $\Delta(k)$.

Since every vertex $v$ of $\Delta(k)$ has $v_{i}=0$ or $v_{i}=1$, at most one of the facets $x_{i} \geq 0$ or $x_{i} \leq 1$ can be in $\mathcal{F}$ for every $i \in[n]$ (otherwise $\mathcal{V}$ would be empty). We can partition $[n]$ into $L \cup U \bullet R$ such that $L$ (resp. $U$ ) contains those indices $i \in[n]$ such that the facet corresponding to $x_{i} \geq 0$ (resp. $x_{i} \leq 1$ ) is in $\mathcal{F}$ and $R$ contains the remaining indices. Lemma 3.2 yields

$$
\mathcal{V}=\left\{v \text { vertex of } \Delta(k): v_{L}=\mathbb{1}, v_{U}=\mathbb{O}\right\}
$$

We now prove that a node set $\mathcal{V}$ of this form is proper $\Lambda$-closed only if $|\mathcal{V}|=1$. Then, Corollary 3.6 yields the claim.

Indeed, if we have $|\mathcal{V}|>1$, then there exist vertices $u, w \in \mathcal{V}$ and indices $i, j \in R$ such that $u_{i}=w_{j}=1, u_{j}=w_{i}=0$, and $u_{l}=w_{l}$ for all $l \notin\{i, j\}$ (see Figure 4). Choose any $s \in L \cup U$ and observe that, since $u, w \in \mathcal{V}, u_{s}=w_{s}=1$ if $s \in L$ and $u_{s}=w_{s}=0$ if $s \in U$. The following vertex is easily checked to be adjacent to $u$ and $w$ (min and max must be read component-wise):

$$
v:= \begin{cases}\max (u, w)-\mathbb{e}^{s} & \text { if } s \in L \\ \min (u, w)+\mathbb{e}^{s} & \text { if } s \in U\end{cases}
$$




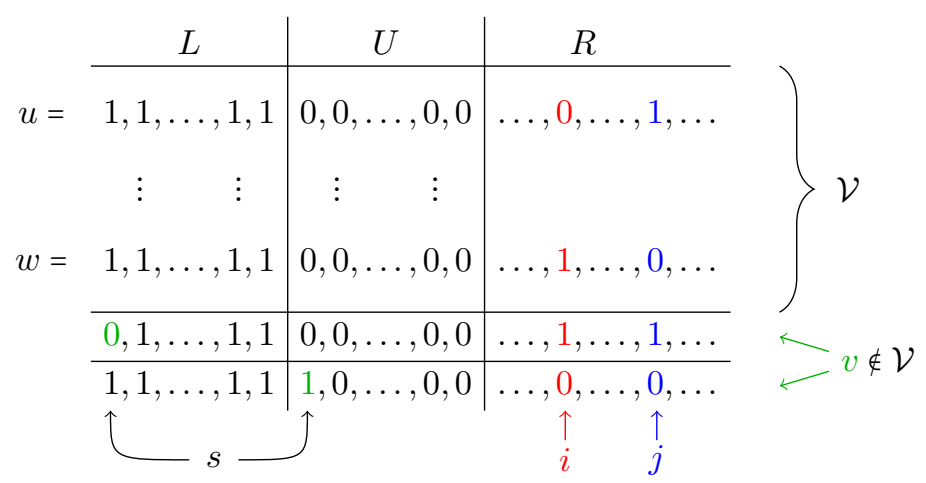

Figure 4: Vertices of $\Delta(k)$ in $\mathcal{V}$ for a Biclique.

As $v_{s}=0$ if $s \in L$ and $v_{s}=1$ if $s \in U, v \notin \mathcal{V}$. This contradicts the fact that $\mathcal{V}$ is $\Lambda$-closed.

\section{Spanning Tree Polytope}

In this section we bound the simple extension complexity of the spanning tree polytope $P_{\text {spt }}\left(K_{n}\right)$ of the complete graph $K_{n}$ with $n$ nodes. In order to highlight different perspectives we mention three equivalent adjacency characterizations which all follow from the fact that the spanning tree polytope is the base polytope of a graphic matroid (see [22], Theorem 40.6.). The vertices corresponding to spanning trees $T$ and $T^{\prime}$ are adjacent in the spanning tree polytope if and only if ...

- ... $\left|T \Delta T^{\prime}\right|=2$ holds.

- ... $T^{\prime}$ arises from $T$ by removing one edge and reconnecting the two connected components by another edge.

- ... $T^{\prime}$ arises from $T$ by adding one additional adge and removing any edge from the cycle that this edge created.

From the third statement it is easy to see that the maximum degree of the 1skeleton of $P_{\text {spt }}\left(K_{n}\right)$ is in $\mathcal{O}\left(n^{3}\right)$, since there are $\mathcal{O}\left(n^{2}\right)$ possible choices for the additional edge, each of which yields $\mathcal{O}(n)$ choices for a cycle-edge to remove.

Lemma 5.1. All proper $\Lambda$-closed sets in the graph of $P_{s p t}\left(K_{n}\right)$ are isolated.

Proof. Throughout the proof, we will identify vertices with the corresponding spanning trees.

Suppose $\mathcal{V}$ is a proper $\Lambda$-closed set that is not isolated. Then there are spanning trees $T_{1}, T_{2} \in \mathcal{V}$ and $T_{3} \notin \mathcal{V}$, such that $T_{1}$ is adjacent to both $T_{2}$ and $T_{3}$, but $T_{2}$ and $T_{3}$ are not adjacent.

Let $e$ be the unique edge which is in $T_{1}$ but not in $T_{2}$, i.e., $\{e\}=T_{1} \backslash T_{2}$. Analogously, let $\{f\}=T_{2} \backslash T_{1},\{g\}=T_{1} \backslash T_{3}$, and $\{h\}=T_{3} \backslash T_{1}$. Since $T_{2}$ and $T_{3}$ are not adjacent in the polytope, their symmetric difference $T_{2} \Delta T_{3} \subseteq\{e, f, g, h\}$ 


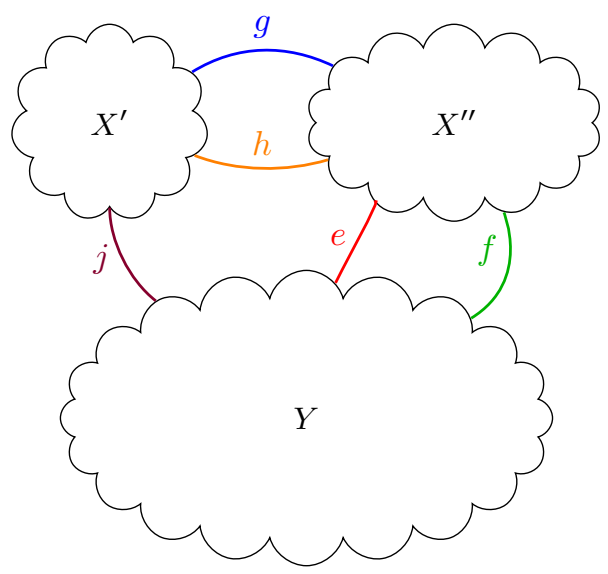

Figure 5: Case 2 of Lemma 5.1.

must have cardinality greater than 2 . Because the symmetric difference of two spanning trees consists of an even number of edges, that cardinality must be equal to 4 , proving $e \neq g$. Let us define $F$ by $F=T_{1} \backslash\{e, g\}$ which is a tree with two edges missing, i.e., a forest with three connected components $X^{\prime}, X^{\prime \prime}, Y$. W.l.o.g., $g$ connects $X^{\prime}$ with $X^{\prime \prime}$ and $e$ connects $X^{\prime \prime}$ with $Y$. In their turn, $T_{2}$ and $T_{3}$ can be written as $F \cup\{f, g\}$ and $F \cup\{e, h\}$, respectively.

There are two possible cases for $h$ :

Case 1: $h$ connects $Y$ with $X^{\prime}$ or $X^{\prime \prime}$.

Let $T^{\prime}:=F \cup\{g, h\}$ and observe that $T^{\prime}$ is a spanning tree since $g$ connects $X^{\prime}$ with $X^{\prime \prime}$ and $h$ connects one of both with $Y$. Obviously, $T^{\prime}$ is adjacent to $T_{1}, T_{2}$, and $T_{3}$. Since $T^{\prime}$ is adjacent to $T_{1}$ and $T_{2}, T^{\prime} \in \Lambda(\mathcal{V})=\mathcal{V}$. Since $T_{3}$ is adjacent to $T_{1}, T^{\prime} \in \mathcal{V}$, this in turn implies the contradiction $T_{3} \in \mathcal{V}$.

Case 2: $h$ connects $X^{\prime}$ with $X^{\prime \prime}$.

Let $j$ be any edge connecting $X^{\prime}$ with $Y$ (recall that we dealing with a complete graph) and let $T^{\prime}:=F \cup\{g, j\}$ which is a spanning tree adjacent to $T_{1}$ and $T_{2}$ and hence $T^{\prime} \in \Lambda(\mathcal{V})=\mathcal{V}$. Clearly, $T^{\prime \prime}:=F \cup\{e, j\}$ is a spanning tree adjacent to $T_{1}$ and $T^{\prime}$ and hence $T^{\prime \prime} \in \mathcal{V}$. Finally, let $T^{\prime \prime \prime}:=F \cup\{h, j\}$ be a third spanning tree adjacent to $T^{\prime}$ and $T^{\prime \prime}$. Again, we have $T^{\prime \prime \prime} \in \mathcal{V}$ due to $\Lambda(\mathcal{V})=\mathcal{V}$.

Since $T_{3}$ is adjacent to $T_{1}$ and $T^{\prime \prime \prime}$, exploiting $\Lambda(\mathcal{V})=\mathcal{V}$ once more yields the contradiction $T_{3} \in \mathcal{V}$.

Using this result we immediately get a lower bound of $\Omega\left(n^{3}\right)$ for the simple extension complexity of $P_{\mathrm{spt}}\left(K_{n}\right)$ since the maximum degree of its graph is of that order. However, we can prove a much stronger result.

Theorem 5.2. The simple extension complexity of the spanning tree polytope of $K_{n}$ is in $\Omega\left(2^{n-o(n)}\right)$.

Proof. Assume $n \geq 5$ and let $s, t$ be any two distinct nodes of $K_{n}$. Consider the following set of subsets of the nodes $V \backslash\{s, t\}$

$$
\mathcal{W}:=\{W \subseteq V \backslash\{s, t\}:|W|=\lfloor n / 2\rfloor\} .
$$




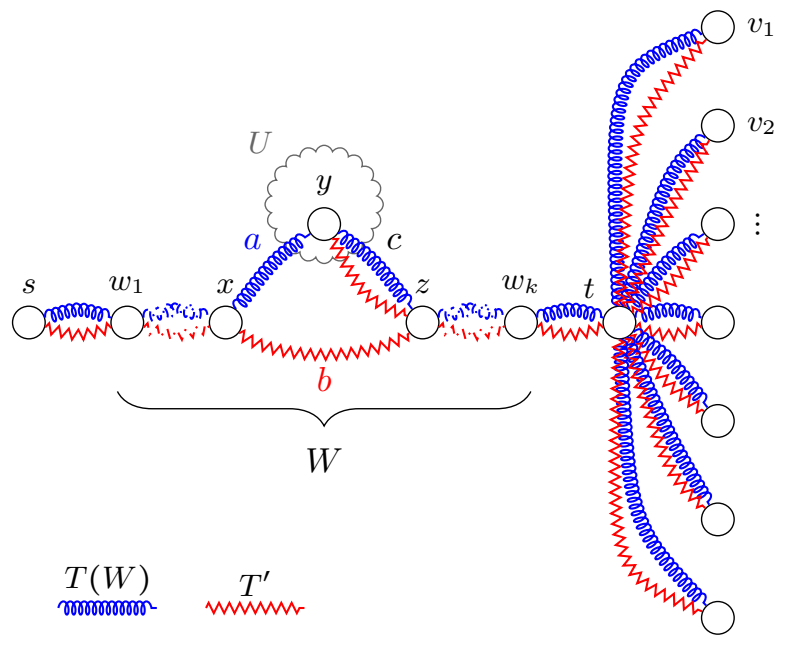

Figure 6: Construction for Theorem 5.2.

Let $k:=\lfloor n / 2\rfloor$, fix some ordering of the nodes $w_{1}, w_{2}, \ldots, w_{k} \in W$ for each $W \in \mathcal{W}$ and define a specific tree $T(W)$

$$
\begin{aligned}
T(W):= & \left\{\left\{s, w_{1}\right\},\left\{w_{k}, t\right\}\right\} \\
& \cup\left\{\left\{w_{i}, w_{i+1}\right\}: i \in[k-1]\right\} \\
& \cup\{\{t, v\}: v \notin(W \cup\{s, t\})\}
\end{aligned}
$$

as depicted in Figure 6. We will now prove that for each simple extension of $P_{\text {spt }}\left(K_{n}\right)$ every such $T(W)$ must be in a different induced vertex set.

Let $W \in \mathcal{W}$ be some set $W$ with tree $T(W)$. Let $\mathcal{F}$ and $\mathcal{V}$ be the face and vertex sets, respectively, induced by a proper facet of a simple extension such that $T(W)$ is in $\mathcal{V}$. Construct an adjacent tree $T^{\prime}$ as follows.

Choose some vertex $y \in W$ and let $x-y-z$ be a subpath of the $s$-t-path in $T(W)$ in that order. Note that $\{x, y, z\} \subseteq W \cup\{s, t\}$. Denote by $a, b, c$ the edges $\{x, y\},\{x, z\}$, and $\{y, z\}$, respectively.

Let $T^{\prime}=T(W) \backslash\{a\} \cup\{b\}$. Because $T^{\prime}$ is adjacent to $T(W)$, by Lemma 5.1 we know $T^{\prime} \notin \mathcal{V}$. Hence, due to Lemma 3.2 , there must be a facet $F \in \mathcal{F}$ defined by $x(E[U]) \leq|U|-1$ (with $|U| \geq 2$ ) which contains $T^{\prime}$. Furthermore, this facet does not contain $T(W)$ because $T(W) \in \mathcal{V}$ holds. Hence, we have $|T(W)[U]|<|U|-1$ and $\left|T^{\prime}[U]\right|=|U|-1$. This implies $|T(W) \cap \delta(U)| \geq 2$ and $\left|T^{\prime} \cap \delta(U)\right|=1$. Obviously, $a \in \delta(U)$ and $b \notin \delta(U)$.

Then $x, z \in U$ if and only if $y \notin U$ because $a \in \delta(U)$ and $b \notin \delta(U)$. Hence, $c \in \delta(U)$, i.e., $T \cap \delta(U)=\{c\}$. Due to $|U| \geq 2$, this implies $U=V \backslash\{y\}$.

As this can be argued for any $y \in W$, we have that the facets defined by $V \backslash\{y\}$ are in $\mathcal{F}$ for all $y \in W$. Hence, $\mathcal{V}$ contains only trees $T$ for which $|T \cap \delta(V \backslash\{y\})|=|T \cap \delta(\{y\})| \geq 2$, i.e., no leaf of $T$ is in $W$.

This shows that for distinct sets $W, W^{\prime} \in \mathcal{W}$, any vertex set $\mathcal{V}$ induced by a proper facet of a simple extension that contains $T(W)$ does not contain $T\left(W^{\prime}\right)$ because any vertex $v \in W \backslash W^{\prime}$ is a leaf of $T\left(W^{\prime}\right)$. Hence, the number of simple 
bicliques is at least

$$
|\mathcal{W}|=\left(\begin{array}{c}
n-2 \\
\lfloor n / 2\rfloor
\end{array}\right) \in \Omega\left(2^{n-o(n)}\right)
$$




\section{Flow Polytopes for Acyclic Networks}

Many extended formulations model the solutions to the original formulation via a path in a specifically constructed directed acyclic graph. A simple example is the linear-size formulation for the parity polytope by Carr and Konjevod [5], and a more elaborate one is the approximate formulation for 0/1-knapsack polytopes by Bienstock [4].

Let $D=(V, A)$ be a directed acyclic graph with fixed source $s \in V$ and sink $t \in V$. By $\mathcal{P}_{s, t}(D)$ we denote the arc-sets of $s$ - $t$-paths in $D$. For some path $P \in \mathcal{P}_{s, t}(D)$ and nodes $u, v \in V(P)$, we denote by $\left.P\right|_{(u, v)}$ the subpath of $P$ going from $u$ to $v$.

For acyclic graphs, the convex hull of the characteristic vectors of all $s$ - $t$ paths is equal to the uncapacitated $s$-t-flow polytope $P_{\text {s-t-flow }}(D)$ with flowvalue 1 , since the linear description of the latter is totally unimodular. The inequalities in this description correspond to nonnegativity constraints of the arc variables, and a vertex corresponding to the path $P$ is obviously non-incident to a facet corresponding to $y_{a} \geq 0$ if and only if $a \in P$ holds. Adjacency in the path polytope was characterized by Gallo and Sodini [10] and can be stated as follows: Two $s$ - $t$-paths $P, P^{\prime}$ correspond to adjacent vertices of the polytope if and only if their symmetric difference consists of two paths from $x$ to $y(x, y \in V$, $x \neq y$ ) without common inner nodes. In other words, they must split and merge exactly once.

Such a network formulation can be easily decomposed into two independent formulations if a node $v$ exists such that every $s$ - $t$-path traverses $v$. We are now interested in the simple extension complexities of flow polytopes of $s$-t-networks that cannot be decomposed in such a trivial way. Our main result in this section is the following:

Theorem 6.1. Let $D=(V, A)$ be a directed acyclic graph with source $s \in V$ and sink $t \in V$ such that for every node $v \in V \backslash\{s, t\}$ there exists an $s$-t-path in $D$ which does not traverse $v$.

Then the simple extension complexity of $P_{s-t-f l o w}(D) \subseteq \mathbb{R}_{+}^{A}$ is equal to the number of distinct $s$-t-paths $\left|\mathcal{P}_{s, t}(D)\right|$.

Proof. Let $\mathcal{F}$ and $\mathcal{V}$ be the face and vertex sets induced by a proper facet of a simple extension of $P_{\text {s-t-flow }}(D)$, respectively. The goal is to prove $|\mathcal{V}|=1$, let us assume for the sake of contradiction $|\mathcal{V}| \geq 2$. By Theorem 3.3 (b), the (inclusion-wise) maximal faces in $\mathcal{F}$ are facets. Let $\varnothing \neq B^{\prime} \subseteq A$ be the arc set corresponding to these facets. By Lemma 3.2, $\mathcal{V}$ is the set of (characteristic vectors of) paths $P \in \mathcal{P}_{s, t}(D)$ satisfying $P \supseteq B^{\prime}$. Let $B \subseteq A$ be the set of arcs common to all such paths and note that $B \supseteq B^{\prime} \neq \varnothing$.

By construction, for any path $P \in \mathcal{V}$ and any arc $a \in P \backslash B$, there is an alternative path $P^{\prime} \in \mathcal{V}$ with $a \notin P^{\prime}$.

Let us fix one of the paths $P \in \mathcal{V}$. Let, without loss of generality, $\left(x^{\prime}, x\right) \in B$ be such that the arc of $P$ leaving $x$ (exists and) is not in $B$. If such an arc does not exist, since $B \neq P$, there must be an arc $\left(x, x^{\prime}\right) \in B$ such that the arc of $P$ entering $x$ is not in $B$. In this case, revert the directions of all arcs in $D$ and exchange the roles of $s$ and $t$ and apply subsequent arguments to the new network. Let $y$ be the first node on $\left.P\right|_{(x, t)}$ different from $x$ and incident to some arc in $B$ or, if no such $y$ exists, let $y:=t$. Paths in $\mathcal{V}$ must leave $x$ and enter $y$ 
but may differ inbetween. The set of traversed nodes is defined as

$$
S:=\{v \in V \backslash\{x, y\}: \exists x-v-y \text {-path in } D\} .
$$

By construction, $x \notin\{s, t\}$ and by the assumptions of the Theorem there exists a path $P^{\prime} \in \mathcal{P}_{s, t}(D)$ which does not traverse $x$. Let $s^{\prime}$ be the last node on $\left.P\right|_{(s, x)}$ that is traversed by $P^{\prime}$. Analogously, let $t^{\prime}$ be the first node of $V\left(\left.P\right|_{(x, t)}\right) \cup S$ that is traversed by $P^{\prime}$. Note that $t^{\prime} \neq x$ since $t^{\prime}$ is traversed by $P^{\prime}$ but $x$ is not. We now distinguish two cases for which we show that $\mathcal{V}$ is not $\Lambda$-closed yielding a contradiction to Corollary 3.4:

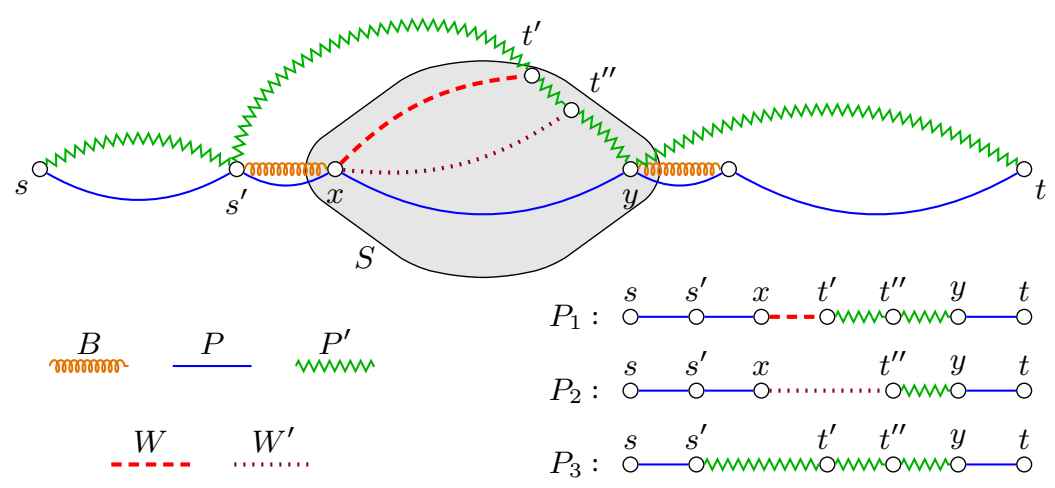

Figure 7: Construction for Case 1 in the Proof of Theorem 6.1.

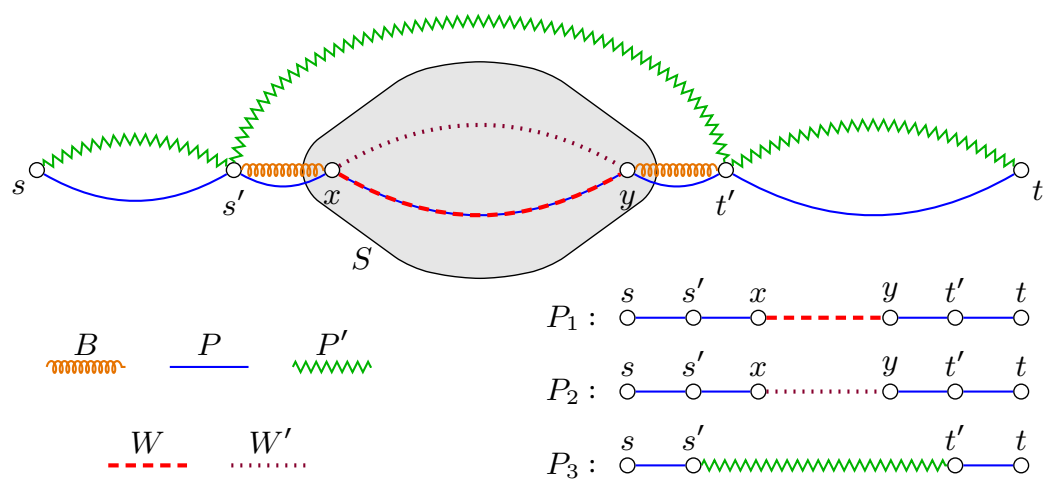

Figure 8: Construction for Case 2 in the Proof of Theorem 6.1.

Case 1: $t^{\prime} \in S$.

By definition of $S$ there must be an $x-t^{\prime}-y$-path $W$. Note that $t^{\prime}$ could be equal to $y$ and then $W$ could agree with $\left.P\right|_{(x, y)}$ as well. Let $\left(z, t^{\prime}\right) \in W$ be the arc of $W$ entering $t^{\prime}$. By definition of $y$, we conclude that $\left(z, t^{\prime}\right) \notin B$. Hence, there is an alternative $x$ - $y$-path $W^{\prime} \neq W$ which does not use $\left(z, t^{\prime}\right)$. We choose $W^{\prime}$ such that it uses as many arcs of $\left.W\right|_{\left(t^{\prime}, y\right)}$ as possible. Construct the following 
three paths (see Figure 7):

$$
\begin{aligned}
& P_{1}:=\left.\left.P\right|_{(s, x)} \cup W \cup P\right|_{(y, t)} \\
& P_{2}:=\left.\left.P\right|_{(s, x)} \cup W^{\prime} \cup P\right|_{(y, t)} \\
& P_{3}:=\left.\left.\left.\left.P\right|_{\left(s, s^{\prime}\right)} \cup P^{\prime}\right|_{\left(s^{\prime}, t^{\prime}\right)} \cup W\right|_{\left(t^{\prime}, y\right)} \cup P\right|_{(y, t)}
\end{aligned}
$$

By construction $P_{1}, P_{2} \in \mathcal{V}$ but $P_{3} \notin \mathcal{V} . P_{1}$ and $P_{3}$ are adjacent in $P_{\text {s-t-flow }}(D)$ since they only differ in the disjoint paths from $s^{\prime}$ to $t^{\prime}$. Analogously, $P_{2}$ and $P_{3}$ are adjacent and thus, contradicting the fact that $\mathcal{V}$ is $\Lambda$-closed.

\section{Case 2: $t^{\prime} \notin S$.}

Let $W:=\left.P\right|_{(x, y)}$ and let $W^{\prime}$ be a different $x$-y-path which must exist by definition of $y$. Construct the following three paths (see Figure 8):

$$
\begin{aligned}
& P_{1}:=P=\left.\left.P\right|_{(s, x)} \cup W \cup P\right|_{(y, t)} \\
& P_{2}:=\left.\left.P\right|_{(s, x)} \cup W^{\prime} \cup P\right|_{(y, t)} \\
& P_{3}:=\left.\left.\left.P\right|_{\left(s, s^{\prime}\right)} \cup P^{\prime}\right|_{\left(s^{\prime}, t^{\prime}\right)} \cup P\right|_{\left(t^{\prime}, t\right)}
\end{aligned}
$$

By construction $P_{1}, P_{2} \in \mathcal{V}$ but $P_{3} \notin \mathcal{V}$ since it does not use $\left(x^{\prime}, x\right) \in B$. $P_{1}$ and $P_{3}$ as well as $P_{2}$ and $P_{3}$ are adjacent in $P_{\text {s-t-flow }}(D)$ since they only differ in the disjoint paths from $s^{\prime}$ to $t^{\prime}$. Again, this contradicts the fact that $\mathcal{V}$ is $\Lambda$-closed.

\section{Perfect Matching Polytope}

The matching polytope and the perfect matching polytope of a graph $G=(V, E)$ are defined as

$$
\begin{aligned}
& P_{\text {match }}(G):=\operatorname{conv}\{\chi(M): M \text { matching in } G\} \\
& P_{\text {match }}^{\text {perf }}(G):=\operatorname{conv}\{\chi(M): M \text { perfect matching in } G\},
\end{aligned}
$$

where $\chi(M) \in\{0,1\}^{E}$ is the characteristic vector of the set $M \subseteq E$, i.e., $\chi(M)_{e}=$ 1 if and only if $e \in M$. We mainly consider the (perfect) matching polytope of the complete graph with $2 n$ nodes $P_{\text {match }}^{\text {perf }}\left(K_{2 n}\right)$. Our main theorem here reads as follows:

Theorem 7.1. The simple extension complexity of the perfect matching polytope of $K_{2 n}$ is equal to its number of vertices $\frac{(2 n) !}{n ! 2^{n}}$.

We first give the high-level proof which uses a structural result presented afterwards.

Proof. The proof is based on Theorem 7.4. It states that for any three perfect matchings $M_{1}, M_{2}, M_{3}$ in $K_{2 n}$, where $M_{1}$ and $M_{2}$ are adjacent (i.e., the corresponding vertices are adjacent), $M_{3}$ is adjacent to both $M_{1}$ and $M_{2}$ or there exists a fourth matching $M^{\prime}$ adjacent to all three matchings.

Let $P=P_{\text {match }}^{\text {perf }}\left(K_{2 n}\right)$ and suppose that $\mathcal{V}$ is a proper $\Lambda$-closed set with $|\mathcal{V}| \geq 2$. Since the polytope's graph is connected there exists a matching $M_{1} \notin \mathcal{V}$ adjacent to some matching $M_{2} \in \mathcal{V}$. Let $M_{3} \in \mathcal{V} \backslash\left\{M_{2}\right\}$. As $\mathcal{V}$ is $\Lambda$-closed and $M_{3} \in \mathcal{V}$ holds, $\left\{M_{1}, M_{2}, M_{3}\right\}$ cannot be a triangle. Hence, by Theorem 7.4 mentioned above, there exists a common neighbor matching $M^{\prime}$. Since $M^{\prime}$ is 
adjacent to $M_{2}$ and $M_{3}$, we conclude $M^{\prime} \in \mathcal{V}$. But now $M_{1} \notin \mathcal{V}$ is adjacent to the two matchings $M_{2}$ and $M^{\prime}$ from $\mathcal{V}$ contradicting the fact that $\mathcal{V}$ is $\Lambda$-closed.

Hence all proper $\Lambda$-closed sets are singletons which implies the claim due to Corollary 3.6.

Since $P_{\text {match }}^{\text {perf }}\left(K_{2 n}\right)$ is a face of $P_{\text {match }}\left(K_{2 n}\right)$ and simple extensions of polytopes induce simple extensions of their faces we obtain the following corollary for the latter polytope.

Corollary 7.2. The simple extension complexity of the matching polytope of $K_{2 n}$ is at least $\frac{(2 n) !}{n ! \cdot 2^{n}}$.

\subsection{Adjacency Result for the Perfect Matching Polytope}

We now turn to the mentioned result on the adjacency structure of the perfect matching polytope of $K_{2 n}$. It is a generalization of the diameter result of Padberg and Rao's in [16].

Clearly, the symmetric difference $M \Delta M^{\prime}$ of two perfect matchings is always a disjoint union of alternating cycles, so-called $M-M^{\prime}$-cycles. Chvátal [6] showed that (the vertices corresponding to) two perfect matchings $M$ and $M^{\prime}$ are adjacent if and only if $M \Delta M^{\prime}$ forms a single alternating cycle. For an edge set $F$ we denote by $V(F)$ the set of nodes covered by the edges of $F$. We start with an easy construction and modify the resulting matching later.
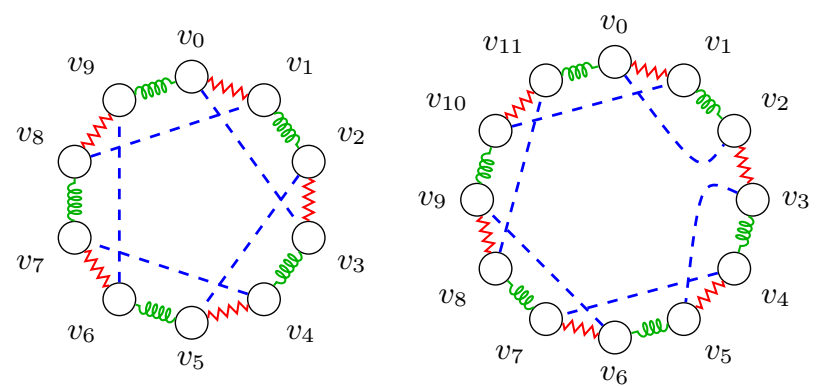

Figure 9: Lemma 7.3 for a 10-cycle and a 12-cycle.

Lemma 7.3. For any adjacent perfect matchings $M_{1}, M_{2}$ there exists a perfect matching $M^{\prime}$ adjacent to $M_{1}$ and $M_{2}$ that satisfies

$$
V\left(M_{1} \Delta M_{2}\right)=V\left(M_{1} \Delta M^{\prime}\right)=V\left(M_{2} \Delta M^{\prime}\right)
$$

and $M^{\prime} \cap\left(M_{1} \Delta M_{2}\right)=\varnothing$.

Proof. Let $v_{0}, v_{1}, \ldots, v_{2 l-1}, v_{2 l}=v_{0}$ be the set of ordered nodes of the cycle $M_{1} \Delta M_{2}$ and identify $v_{2 l+1}=v_{1}$. If $l$ is odd,

$$
M^{\prime}:=\left\{\left\{v_{i}, v_{i+3}\right\}: i=0,2,4,6, \ldots, 2 l-2\right\}
$$


induces $M_{i}-M^{\prime}$-cycles visiting the nodes in the following order:

$$
\begin{aligned}
M_{1} \Delta M^{\prime}: & v_{0}, v_{3}, v_{2}, v_{5}, v_{4}, v_{7}, v_{6}, \ldots, v_{2 l-1}, \\
& v_{2 l-2}, v_{1}, v_{0} \\
M_{2} \Delta M^{\prime}: & v_{0}, v_{3}, v_{4}, v_{7}, v_{8}, \ldots, v_{2 l-3}, v_{2 l-2}, \\
& v_{1}, v_{2}, v_{5}, v_{6}, \ldots, v_{2 l-4}, v_{2 l-1}, v_{0}
\end{aligned}
$$

If $l$ is even,

$$
\begin{aligned}
M^{\prime} & :=\left\{\left\{v_{i}, v_{i+3}\right\}: i=4,6, \ldots, 2 l-2\right\} \\
& \cup\left\{\left\{v_{0}, v_{2}\right\},\left\{v_{3}, v_{5}\right\}\right\}
\end{aligned}
$$

induces $M_{i}-M^{\prime}$-cycles visiting the nodes in the following order:

$$
\begin{aligned}
M_{1} \Delta M^{\prime}: & v_{0}, v_{2}, v_{3}, v_{5}, v_{4}, v_{7}, v_{6}, \ldots, v_{2 l-1}, \\
& v_{2 l-2}, v_{1}, v_{0} \\
M_{2} \Delta M^{\prime}: \quad & v_{0}, v_{2}, v_{1}, v_{2 l-2}, v_{2 l-3}, \ldots, v_{6}, v_{5}, v_{3}, \\
& v_{4}, v_{7}, v_{8}, \ldots, v_{2 l-4}, v_{2 l-1}, v_{0}
\end{aligned}
$$

Figure 9 shows examples for both cases. It is easy to see that the node sets of the cycles equals the node set of $M_{1} \Delta M_{2}$ and that $M^{\prime} \cap\left(M_{1} \Delta M_{2}\right)=\varnothing$ holds. In order to produce a perfect matching on all nodes we simply add $M_{1} \cap M_{2}$ to $M^{\prime}$ which does not change any of the two required properties.

Suppose there is a third perfect matching $M_{3}$ and we want to make $M^{\prime}$ adjacent to this matching as well. The remainder of this section is dedicated to the proof of the following result.

Theorem 7.4. Let $M_{1}$ and $M_{2}$ be two adjacent perfect matchings and $M_{3}$ a third perfect matching. Then the three matchings are pairwise adjacent or there exists a perfect matching $M^{\prime}$ adjacent to all three.

Before we state the proof, we introduce the notion of good perfect matchings. The first part of the proof is dedicated to proving their existence, while the second part shows that good perfect matchings, which are minimal in a certain sense, satisfy the properties claimed by Theorem 7.4.

We first fix some notation for the rest of this section. Let $M_{1}, M_{2}$ and $M_{3}$ be three perfect matchings such that $M_{1}$ and $M_{2}$ are adjacent. Denote by $V^{*}:=V\left(M_{1} \Delta M_{2}\right)$ the node set of the single alternating $M_{1}-M_{2}$-cycle.

For a perfect matching $M^{\prime}$ we denote by $M_{3}-M^{\prime}$-components the connected components of $M_{3} \cup M^{\prime}$ and by $c\left(M_{3}, M^{\prime}\right)$ their number. We call a perfect matching $M^{\prime}$ good if the following five properties hold:

(A) $M^{\prime}$ is adjacent to $M_{1}$ and $M_{2}$.

(B) All $M_{3}-M^{\prime}$-components touch the node-set $V^{*}$ of $M_{1} \Delta M_{2}$.

(C) All $M^{\prime}$-edges which also belong to the $M_{1}-M_{2}$-cycle, i.e., the edges from $M^{\prime} \cap\left(M_{1} \Delta M_{2}\right)$, are contained in the same $M_{3}-M^{\prime}$-component.

(D) $M_{3} \neq M^{\prime}$ and $c\left(M_{3}, M^{\prime}\right) \leq \frac{1}{2}\left|M_{1} \Delta M^{\prime}\right|+\frac{1}{2}\left|M_{2} \Delta M^{\prime}\right|-3$ holds.

(E) $c\left(M_{3}, M^{\prime}\right) \leq \frac{1}{2}\left|M_{j} \Delta M^{\prime}\right|$ holds for $j=1,2$ and equality holds only if we have $V\left(M_{k} \Delta M^{\prime}\right) \supseteq V^{*}$ for $k=3-j$, i.e., $\left\{M_{j}, M_{k}\right\}=\left\{M_{1}, M_{2}\right\}$. 
We first establish the existence of good perfect matchings.

Lemma 7.5. Let $M_{1}, M_{2}, M_{3}$ be three perfect matchings of $K_{2 n}$ such that $M_{1} \cap M_{2} \cap M_{3}=\varnothing$ holds and such that $M_{1}$ and $M_{2}$ are adjacent, but $M_{3}$ is not adjacent to both of them.

Then there exists a good perfect matching $M^{\prime}$.

Proof. Let $\bar{M}$ be the perfect matching adjacent to $M_{1}$ and $M_{2}$ constructed in Lemma 7.3.

Note that it satisfies $\bar{M} \cap\left(M_{1} \Delta M_{2}\right)=\varnothing$ as well as $\left|M_{1} \Delta \bar{M}\right|=\left|M_{2} \Delta \bar{M}\right|=$ $\left|M_{1} \Delta M_{2}\right|=\left|V^{*}\right| \geq 4$. We now enlarge the $M_{i}-\bar{M}$-cycles $(i=1,2)$ in order to remove $M_{3}-\bar{M}$-cycles which do not touch $V^{*}$ in order to satisfy Property (B).

Let $\left\{u_{0}, v_{0}\right\}$ be an $\bar{M}$-edge with $u_{0}, v_{0} \in V^{*}$. Let $C_{1}, C_{2}, \ldots, C_{s}$ be all $M_{3}$ $\bar{M}$-cycles with $V\left(C_{i}\right) \cap V^{*}=\varnothing$ and let, for $i=1,2, \ldots, s,\left\{u_{i}, v_{i}\right\} \in C_{i} \cap \bar{M}$ be any $\bar{M}$-edge of $C_{i}$. Define $M^{\prime}$ to be

$$
\begin{aligned}
M^{\prime}:= & \left(\bar{M} \backslash\left\{\left\{u_{i}, v_{i}\right\}: i=0,1, \ldots, s\right\}\right) \\
& \cup\left\{\left\{u_{i}, v_{i+1}\right\}: i=0,1, \ldots, s\right\}
\end{aligned}
$$

where $v_{s+1}=v_{0}($ see Figure 10).

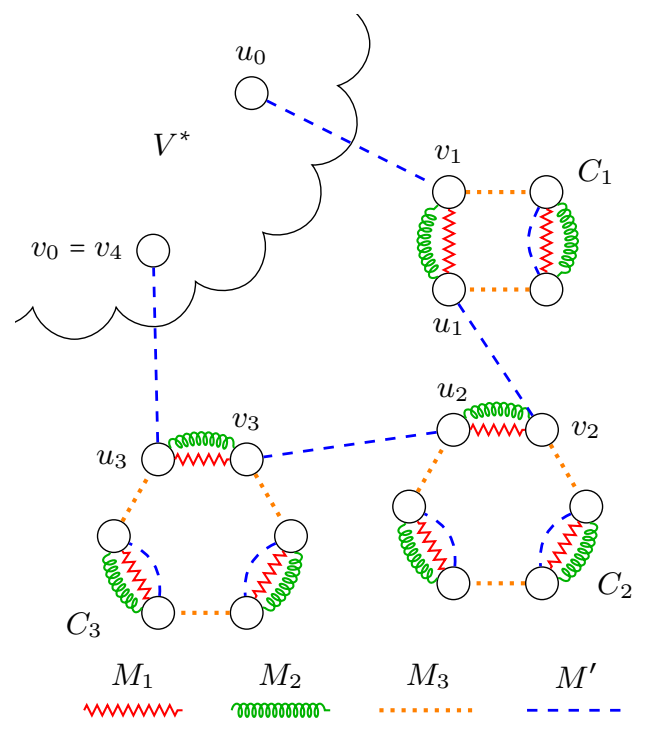

Figure 10: Construction in Lemma 7.5 with 3 outer cycles.

We now verify Property (A), i.e., that $M^{\prime}$ is adjacent to $M_{i}(i=1,2)$. Since the cycles $C_{1}, \ldots, C_{s}$ do not touch $V^{*}, \bar{M}$ and $M_{i}$ coincide outside $V^{*}$. Hence, the modification replaces the $\bar{M}$-edge $\left\{u_{0}, v_{0}\right\}$ by an alternating $\bar{M}-M_{i}$-path from $u_{0}$ to $v_{0}$ which visits exactly 2 nodes of each $C_{i}$, thus indeed $M^{\prime}$ is adjacent to both $M_{1}$ and $M_{2}$.

In order to prove Properties (B) and (E), let us check how the $M_{3}-M^{\prime}$ components look like. The $M_{3}-M^{\prime}$-cycle constructed above contains nodes $u_{0}$ and $v_{0}$. All other $M_{3}-M^{\prime}$-cycles were also $M_{3}-\bar{M}$-cycles, and hence, by definition of the $C_{i}$ above, have at least two nodes of $V^{*}$ in common since one of their 
$\bar{M}$-edges has both endpoints in $V^{*}$. All edges in $M_{3} \cap M^{\prime}$ must also lie in $V^{*}$ since outside $V^{*} \cup V\left(C_{1}\right) \cup \ldots V\left(C_{s}\right)$ the matchings $M^{\prime}, M_{1}$ and $M_{2}$ are the same and $M_{1} \cap M_{2} \cap M_{3}=\varnothing$ holds.

Hence all $M_{3}-M^{\prime}$-components have at least two nodes in $V^{*}$, in particular, Property (B) holds. It also proves the inequality statement of Property (E) because $V\left(M_{j} \Delta M^{\prime}\right) \supseteq V^{*}$ for $j=1,2$. Furthermore, the containment statement is due to Lemma 7.3, since we have $V\left(M_{k} \Delta M^{\prime}\right) \supseteq V\left(M_{k} \Delta \bar{M}\right)=V^{*}$ for $k=1,2$.

In order to verify Property (C), observe that $\bar{M}$ satisfies $\bar{M} \cap\left(M_{1} \Delta M_{2}\right)=\varnothing$. Since all edges in $M^{\prime}$ that were not in $\bar{M}$ have at least one endpoint outside $V^{*}$, we also have $M^{\prime} \cap\left(M_{1} \Delta M_{2}\right)=\varnothing$. Hence, Property (C) is satisfied trivially.

It remains to show that Property (D) holds. Clearly, since $M_{3}$ is adjacent to at most one of $M_{1}, M_{2}$, we have $M_{3} \neq M^{\prime}$. Since we established as part of Property (E), that $c\left(M_{3}, M^{\prime}\right) \leq \frac{1}{2}\left|M_{1} \Delta M^{\prime}\right|$ holds, it suffices to show that $\left|M_{2} \Delta M^{\prime}\right|$ is at least 6 . Suppose, for the sake of contradiction, that this is not the case, i.e., $\left|M_{2} \Delta M^{\prime}\right| \leq 4$ holds, which in turn implies $c\left(M_{3}, M^{\prime}\right) \leq 2$. Also $\left|M_{2} \Delta M^{\prime}\right| \geq 4$ holds since both matchings are adjacent. This implies that we have equality in the containment $V\left(M_{2} \Delta M^{\prime}\right) \supseteq V\left(M_{2} \Delta \bar{M}\right)=V^{*}$, from which we conclude that $s=0$ holds, i.e., $M^{\prime}=\bar{M}$. These properties already prove that the $M^{\prime}$-edges which match the nodes of $V^{*}$ are exactly the two chords of the $M_{1}-M_{2}$-cycle (see Figure 11). It is now easy to verify that then $M_{1}, M_{2}$, and $M_{3}$ must be pairwise adjacent, a contradiction to the assumptions of this lemma.

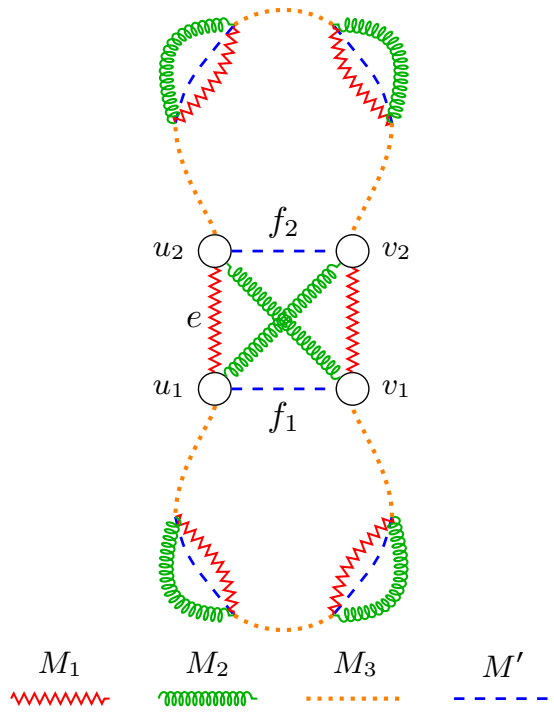

Figure 11: A special case where $M_{3}$ is adjacent to $M_{1}$ and $M_{2}$.

Proof of Theorem 7.4. Let $M_{1}, M_{2}, M_{3}$ be as stated in the Theorem. We assume, without loss of generality, that $M_{1} \cap M_{2} \cap M_{3}=\varnothing$ holds, since otherwise we can restrict ourself to the graph with the nodes of this set deleted. We also assume that $M_{1}, M_{2}$, and $M_{3}$ are not pairwise adjacent, since otherwise there is nothing to prove. 
In this situtation, Lemma 7.5 guarantees the existence of a good perfect matching. Let $M^{\prime}$ be a good perfect matching with minimum $c\left(M_{3}, M^{\prime}\right)$. If $c\left(M_{3}, M^{\prime}\right)=1$ holds, $M^{\prime}$ is adjacent to $M_{3}$ (and also adjacent to $M_{1}$ and $M_{2}$ by Property $(\mathrm{A})$ ) and we are done. Hence, for the sake of contradiction, we from now on assume that $c\left(M_{3}, M^{\prime}\right)$ is at least 2 . The strategy is to construct another good matching $M^{*}$ with $c\left(M_{3}, M^{*}\right)<c\left(M_{3}, M^{\prime}\right)$.

Due to Property (C), there exists an $M_{3}-M^{\prime}$-component $\widehat{C}$ containing all edges (if any) from $M^{\prime} \cap\left(M_{1} \Delta M_{2}\right), M_{1} \Delta M_{2}$ is a single cycle visiting all nodes in $V^{*}$ all of which are in some $M_{3}-M^{\prime}$-component as they are matched by $M^{\prime}$. Thus, by Property (B), the component $\widehat{C}$ is connected to at least one other $M_{3}$ $M^{\prime}$-component by an edge from $M_{1}-M_{2}$. Let us choose such an edge $e \in M_{j} \backslash M_{k}$ for some $j=1,2$ and $k=3-j$, and if such edges exist for both values of $j$, choose $e$ such that $\left|M_{j} \Delta M^{\prime}\right|$ is maximum.

We claim that $\left|M_{j} \Delta M^{\prime}\right| \geq 6$ holds. Suppose, for the sake of contradiction, $\left|M_{j} \Delta M^{\prime}\right|=4$. Property (E) implies that $c\left(M_{3}, M^{\prime}\right) \leq 2$ holds. Since $c\left(M_{3}, M^{\prime}\right) \geq$ 2 also holds, we have equality and then Property (E) implies that the $M_{k}$ $M^{\prime}$-cycle covers all nodes in $V^{*}$. This cycle connects the only two $M_{3}-M^{\prime}$ components $\widehat{C}$ and $C^{\prime}$ via at least two $M_{k}$-edges $f, f^{\prime}$ since the $M^{\prime}$-edges of the cycle are inside their respective components. Note that $\left|M_{k} \Delta M^{\prime}\right| \geq 2 c\left(M_{3}, M^{\prime}\right)+$ $6-\left|M_{j} \Delta M^{\prime}\right| \geq 6$ holds by Property (D). Hence, by the maximality assumption for the choice of edge $e$, this implies that there is no edge in $M_{k} \backslash M_{j}$ which connects $\widehat{C}$ to $C^{\prime}$. Since $f, f^{\prime} \in M_{k}$ both connect $\widehat{C}$ to $C^{\prime}$, it follows that $f, f^{\prime} \in M_{j}$ holds as well. Because $\left|M_{j} \Delta M^{\prime}\right|=4$ holds we have $M_{j} \backslash M^{\prime}=\left\{f, f^{\prime}\right\}$. Hence, the alternating $M_{j}-M^{\prime}$-cycle of length 4 is also an alternating $M_{k}-M^{\prime}$ cycle. But the latter has at least length 6 as argued above which yields a contradiction.

To summarize, we now have two distinct $M_{3}-M^{\prime}$-components $\widehat{C}$ and $C^{\prime}$ connected by an edge $e \in M_{j} \backslash M_{k}$ for some $j=1,2$ such that $\left|M_{j} \Delta M^{\prime}\right| \geq 6$ holds and all edges from $M^{\prime} \cap\left(M_{1} \Delta M_{2}\right)$ (if any) are in $\widehat{C}$.

Let $u_{1} \in V(\widehat{C})$ and $u_{2} \in V\left(C^{\prime}\right)$ be the endpoints of edge $e$. Let $f_{1}=$ $\left\{u_{1}, v_{1}\right\}, f_{2}=\left\{u_{2}, v_{2}\right\} \in M^{\prime}$ be the edges matching $u_{1}$ and $u_{2}$. We clearly have $v_{1} \in V(\widehat{C})$ and $v_{2} \in V\left(C^{\prime}\right)$ as well since $f_{1}$ and $f_{2}$ are contained in their respective $M_{3}-M^{\prime}$-components. In particular we have $f_{1}, f_{2} \neq e$ and $u_{1}, u_{2}, v_{1}, v_{2}$ are pairwise distinct nodes. Because $f_{2} \notin M_{j}\left(e \in M_{j}\right.$ and $\left.e \cap f_{2} \neq \varnothing\right)$ and $f_{2} \nsubseteq V(\widehat{C})$ hold, Property (C) implies $f_{2} \notin M_{k}$ (note that $\widehat{C}$ is the component mentioned in Property $(\mathrm{C})$ ).

If also $f_{1} \notin M_{k}$ holds, $f_{1}$ and $f_{2}$ belong to $M_{k} \Delta M^{\prime}$ which is a single cycle by Property (A), and hence there exists a walk $W$ on $M_{k} \Delta M^{\prime}$ starting in $u_{1}$ with edge $f_{1}$ which visits nodes $u_{2}$ and $v_{2}$ (in some order).

We are now ready to create a new good perfect matching $M^{*}$ related to $M^{\prime}$ by small changes. For this, we distinguish two cases. For each case we establish Property (A) separately, and afterwards prove the remaining properties for both cases in parallel.

Case 1: $f_{1} \notin M_{k}$ holds and $u_{2}$ comes before $v_{2}$ on walk $W$.

Let $M^{*}:=\left(M^{\prime} \backslash\left\{f_{1}, f_{2}\right\}\right) \cup\left\{\left\{u_{1}, u_{2}\right\},\left\{v_{1}, v_{2}\right\}\right\}$ (see Figure 12).

We now prove Property (A) for $M^{*}$. The symmetric difference $M_{k} \Delta M^{*}$ consists of a single cycle that arises from the cycle $M_{k} \Delta M^{\prime}$ by removing edges $f_{1}, f_{2}$ and adding edges $\left\{u_{2}, u_{1}\right\}$ and $\left\{v_{2}, v_{1}\right\}$. For $M_{j}$, the situation is different, 
since there is a new component $e \in M_{j} \cap M^{*}$. The new $M_{j}$ - $M^{*}$-cycle is now 2 edges shorter than the $M_{j}-M^{\prime}$-cycle was before since it visits the edge $\left\{v_{1}, v_{2}\right\}$ instead of the path $v_{1}-u_{1}-u_{2}-v_{2}$. But because we ensured $\left|M_{j} \Delta M^{\prime}\right| \geq 6$ before, we have $\left|M_{j} \Delta M^{*}\right| \geq 4$, that is, $M^{*}$ is also adjacent to $M_{j}$.

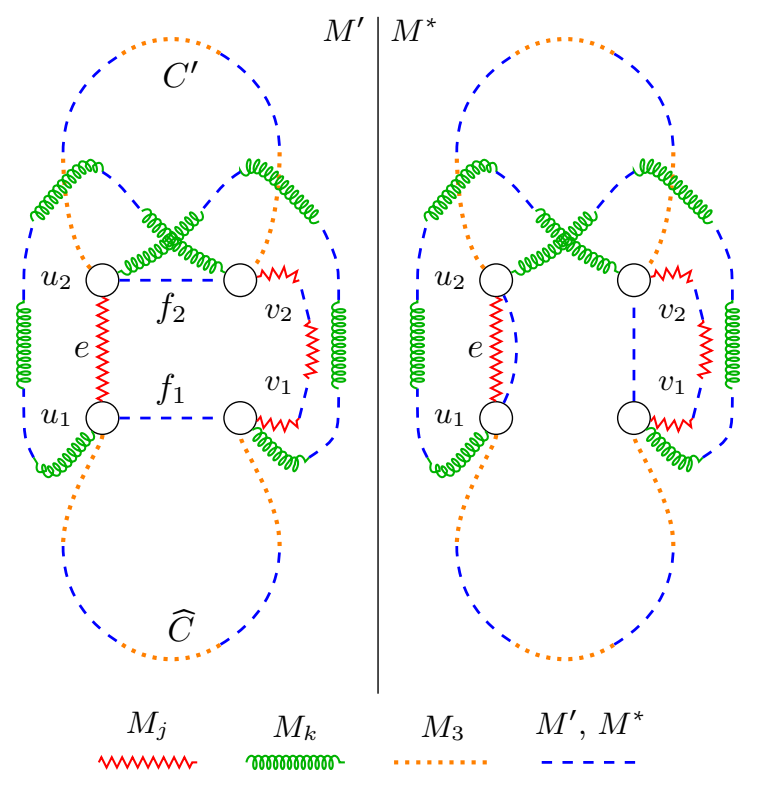

Figure 12: Modifications in Case 1.

Case 2: $f_{1} \in M_{k}$ holds or $f_{1} \notin M_{k}$ and $u_{2}$ comes after $v_{2}$ on walk $W$.

Let $M^{*}:=\left(M^{\prime} \backslash\left\{f_{1}, f_{2}\right\}\right) \cup\left\{\left\{u_{1}, v_{2}\right\},\left\{u_{2}, v_{1}\right\}\right\}$ (see Figure 13).

We now prove Property (A) for $M^{*}$. The symmetric difference $M_{k} \Delta M^{*}$ consists of a single cycle that arises from the cycle $M_{k} \Delta M^{\prime}$ by removing edges $f_{1}, f_{2}$ and adding edges $\left\{v_{2}, u_{1}\right\}$ and $\left\{u_{2}, v_{1}\right\}$. There is also only one $M_{j}-M^{*}$ cycle which is essentially equal to the $M_{j}-M^{\prime}$-cycle, except that the path $v_{1}-$ $u_{1}-u_{2}-v_{2}$ was replaced by the path $v_{1}-u_{2}-u_{1}-v_{2}$. 


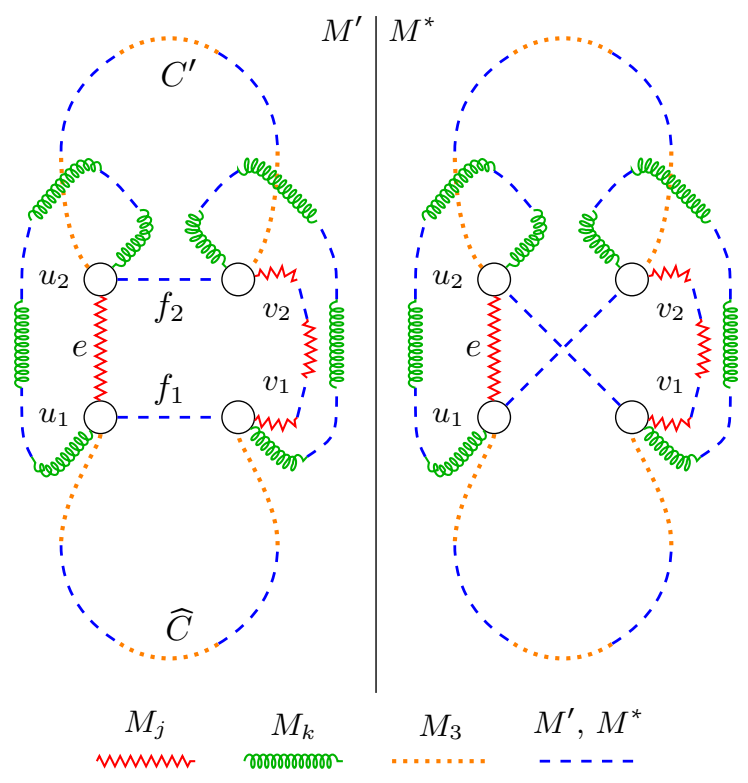

Figure 13: Modifications in Case 2.

In Case 1 as well as in Case $2, M^{*}$ is again a perfect matching since $M^{\prime}$ was a perfect matching and they differ only in the way the nodes $u_{1}, u_{2}, v_{1}, v_{2}$ are matched. Furthermore, $M^{*}$ connects the two components $\widehat{C}$ and $C^{\prime}$, that is, $c\left(M_{3}, M^{*}\right)=c\left(M_{3}, M^{\prime}\right)-1$. In order to create the desired contradiction to the minimality of $c\left(M_{3}, M^{\prime}\right)$, it remains to prove that $M^{*}$ satisfies Properties (B), (C), (D) and (E).

Property (B) is satisfied for $M^{*}$ because $V(\widehat{C})$ is contained in an $M_{3}-M^{*}$ component and all edges in $M^{*} \backslash M^{\prime}$ are contained in the same component.

Property (C) is also satisfied for $M^{*}$ since all $M^{*}$-edges that were not $M^{\prime}$ edges before, are contained in cycle $\widehat{C}$ (which was by definition the only $M_{3}$ $M^{\prime}$-cycle containing edges in $\left.M^{\prime} \cap\left(M_{1} \Delta M_{2}\right)\right)$.

We now prove that Property (D) is satisfied for $M^{*}$. We have

$$
\begin{aligned}
c\left(M_{3}, M^{*}\right)+3 & =c\left(M_{3}, M^{\prime}\right)+3-1 \leq \frac{1}{2}\left|M_{1} \Delta M^{\prime}\right|+\frac{1}{2}\left|M_{2} \Delta M^{\prime}\right|-1 \\
& =\frac{1}{2}\left|M_{k} \Delta M^{\prime}\right|+\frac{1}{2}\left|M_{j} \Delta M^{\prime}\right|-1 \\
& \leq \frac{1}{2}\left|M_{k} \Delta M^{*}\right|+\frac{1}{2}\left(\left|M_{j} \Delta M^{*}\right|+2\right)-1 \\
& =\frac{1}{2}\left|M_{1} \Delta M^{*}\right|+\frac{1}{2}\left|M_{2} \Delta M^{*}\right|,
\end{aligned}
$$

where the first inequality is due to Property (D) for $M^{\prime}$ and the last inequality comes from the fact that in Case $1, M_{j} \Delta M^{*}$ has two fewer edges than $M_{j} \Delta M^{\prime}$ and in Case 2, the cardinalities agree.

By similar arguments, $c\left(M_{3}, M^{*}\right) \leq \frac{1}{2}\left|M_{i} \Delta M^{*}\right|$ holds for $i=1,2$. In order to prove that Property (E) is satisfied for $M^{*}$, assume that $c\left(M_{3}, M^{*}\right)=$ 
$\frac{1}{2}\left|M_{i} \Delta M^{*}\right|$ holds for some $i \in\{1,2\}$. Due to $c\left(M_{3}, M^{*}\right)=c\left(M_{3}, M^{\prime}\right)-1$, this implies $i=j$ and we are in Case 1 since only there $\left|M_{i} \Delta M^{*}\right|$ is less than $\left|M_{i} \Delta M^{\prime}\right|$, and also have $c\left(M_{3}, M^{\prime}\right)=\frac{1}{2}\left|M_{j} \Delta M^{\prime}\right|$. Property (E) of $M^{\prime}$ guarantees that $V\left(M_{k} \Delta M^{\prime}\right) \supseteq V^{*}$ holds. But since the node sets of $M_{k} \Delta M^{\prime}$ and $M_{k} \Delta M^{*}$ are the same, we also have $V\left(M_{k} \Delta M^{*}\right) \supseteq V^{*}$.

We proved that $M^{*}$ is a good perfect matching, yielding the required contradiction to the minimality assumption of $c\left(M_{3}, M^{\prime}\right)$ which completes the proof.

\section{A Related Question}

Let us make a brief digression on the potential relevance of simple extensions with respect to questions related to the diameter of a polytope, i.e., the maximum distance (minimum number of edges on a path) between any pair of vertices in the graph of the polytope. We denote by $\Delta(d, m)$ the maximum diameter of any $d$-dimensional polytope with $m$ facets. It is well-known that $\Delta(d, m)$ is attained by simple polytopes. A necessary condition for a polynomial time variant of the simplex-algorithm to exist is that $\Delta(d, m)$ is bounded by a polynomial in $d$ and $m$ (thus by a polynomial in $m$ ). In fact, in 1957 Hirsch even conjectured (see [7]) that $\Delta(d, m) \leq m-d$ holds, which has only rather recently been disproved by Santos [21]. However, still it is even unknown whether $\Delta(d, m) \leq 2 m$ holds true, and the question, whether $\Delta(d, m)$ is bounded polynomially (i.e., whether the polynomial Hirsch-conjecture is true) is a major open problem in Discrete Geometry.

In view of the fact that linear optimization over a polytope can be performed by linear optimization over any of its extensions, a reasonable relaxed version of that question might be to ask whether every $d$-dimensional polytope $P$ with $m$ facets admits an extension whose size and diameter both are bounded polynomially in $m$. Stating the relaxed question in this naive way, the answer clearly is positive, as one may construct an extension by forming a pyramid over $P$ (after embedding $P$ into $\mathbb{R}^{\operatorname{dim}(P)+1}$ ), which has diameter two. However, in some accordance with the way the simplex algorithm works by pivoting between bases rather than only by proceeding along edges, it seems to make sense to require the extension to be simple (which a pyramid, of course, in general is not). But still, this is not yet a useful variation, since our result on flow polytopes shows that there are polytopes that even do not admit a polynomial (in the number of facets) size simple extension at all. Therefore, we propose to investigate the following question, whose positive answer would be implied by establishing the polynomial Hirsch-conjecture (as every polytope is an extension of itself).

Question 8.1. Does there exist a polynomial $q$ such that every simple polytope $P$ with $m$ facets has a simple extension $Q$ with at most $q(m)$ many facets and diameter at most $q(m)$ ?

Acknowledgements. We are greatful to the referees whose comments lead to significant improvements in the presentation of the material. 


\section{References}

[1] David Avis and Hans Raj Tiwary. On the extension complexity of combinatorial polytopes. In Fedor V. Fomin, Rusins Freivalds, Marta Z. Kwiatkowska, and David Peleg, editors, Automata, Languages, and Programming, volume 7965 of Lecture Notes in Computer Science, pages 57-68. Springer Berlin Heidelberg, 2013.

[2] Egon Balas. Disjunctive programming: Properties of the convex hull of feasible points. MSRR 348, Carnegie Mellon University, 1974.

[3] Egon Balas. Disjunctive programming. In Discrete Optimisation, volume II, pages 3-51, Amsterdam, Netherlands, 1979.

[4] Daniel Bienstock. Approximate formulations for 0-1 knapsack sets. Operations Research Letters, 36(3):317 - 320, 2008.

[5] Robert D. Carr and Goran Konjevod. Polyhedral combinatorics. In H. J. Greenberg, editor, Tutorials on Emerging Methodologies and Applications in Operations Research, volume 76 of International Series in Operations Research \& Management Science, chapter 2, pages 1-46. Springer, 2005.

[6] Vasek Chvátal. On certain polytopes associated with graphs. J. Combin. Theory Ser. B, 18(2):138 - 154, 1975.

[7] G.B. Dantzig. Linear Programming and Extensions. Princeton landmarks in mathematics and physics. Princeton University Press, 1963.

[8] Samuel Fiorini, Volker Kaibel, Kanstantsin Pashkovich, and Dirk Oliver Theis. Combinatorial bounds on nonnegative rank and extended formulations. Discrete Math., 313(1):67-83, 2013.

[9] Samuel Fiorini, Serge Massar, Sebastian Pokutta, Hans Raj Tiwary, and Ronald de Wolf. Linear vs. semidefinite extended formulations: exponential separation and strong lower bounds. In Howard J. Karloff and Toniann Pitassi, editors, STOC, pages 95-106. ACM, 2012.

[10] G. Gallo and C. Sodini. Extreme points and adjacency relationship in the flow polytope. Calcolo, 15:277-288, 1978. 10.1007/BF02575918.

[11] Rafael Gillmann. 0/1-Polytopes Typical and Extremal Properties. PhD thesis, Technische Universität Berlin, 2007.

[12] Michel Goemans. Smallest compact formulation for the permutahedron. http://www-math.mit.edu/ goemans/publ.html, 2009.

[13] Volker Kaibel and Kanstantsin Pashkovich. Constructing extended formulations from reflection relations. In O. Günlük and G. Woeginger, editors, Integer Programming and Combinatorial Optimization. Proceedings of IPCO XV, New York, NY, volume 6655 of Lecture Notes in Computer Science, pages 287-300. Springer, 2011.

[14] Volker Kaibel, Kanstantsin Pashkovich, and Dirk Oliver Theis. Symmetry matters for sizes of extended formulations. SIAM J. Disc. Math., 26(3):1361-1382, 2012. 
[15] R. Kipp Martin. Using separation algorithms to generate mixed integer model reformulations. Oper. Res. Lett., 10(3):119-128, 1991.

[16] Manfred W. Padberg and M. R. Rao. The travelling salesman problem and a class of polyhedra of diameter two. Math. Program., 7:32-45, 1974. 10.1007/BF01585502.

[17] Kanstantsin Pashkovich. Tight lower bounds on the sizes of symmetric extensions of permutahedra and similar results. Mathematics of Operations Research, 39(4):1330-1339, 2014.

[18] Sebastian Pokutta and Mathieu Van Vyve. A note on the extension complexity of the knapsack polytope. Oper. Res. Lett., 41(4):347-350, 2013.

[19] Thomas Rothvoss. The matching polytope has exponential extension complexity. In Proceedings of the 46th Annual ACM Symposium on Theory of Computing, STOC '14, pages 263-272, New York, NY, USA, 2014. ACM.

[20] Thomas Rothvoß. Some 0/1 polytopes need exponential size extended formulations. Math. Program., pages 1-14, 2012.

[21] Francisco Santos. A counterexample to the hirsch conjecture. Annals of Mathematics. Second Series, 176(1):383-412, 2012.

[22] A. Schrijver. Combinatorial Optimization - Polyhedra and Efficiency. Springer, 2003.

[23] Mihalis Yannakakis. Expressing combinatorial optimization problems by linear programs. J. Comput. Syst. Sci., 43(3):441-466, 1991.

[24] G. M. Ziegler. Lectures on Polytopes (Graduate Texts in Mathematics). Springer, 2001. 